\title{
Evidence-based clinical practice guidelines for Crohn's disease, integrated with formal consensus of experts in Japan
}

\author{
Fumiaki Ueno - Toshiyuki Matsui - Takayuki Matsumoto Katsuyoshi Matsuoka - Mamoru Watanabe • \\ Toshifumi Hibi - On Behalf of the Guidelines Project Group of the Research Group of Intractable \\ Inflammatory Bowel Disease subsidized by the Ministry of Health, Labour and Welfare of Japan \\ and the Guidelines Committee of the Japanese Society of Gastroenterology
}

Received: 14 August 2012 / Accepted: 16 August 2012/Published online: 23 October 2012

(C) The Author(s) 2012. This article is published with open access at Springerlink.com

\begin{abstract}
Crohn's disease is a disorder of unknown etiology and complicated pathogenesis. A substantial amount of evidence has accumulated recently and has been applied to clinical practice. The present guidelines were developed based on recent evidence and the formal consensus of experts relevant to this disease. Here we provide an overview of these guidelines, as follows.

- Target disease: Crohn's disease

- Users: Clinical practitioners in internal medicine, surgery, gastroenterology, and general practice

- Purpose: To provide appropriate clinical indicators to practitioners

- Scope of clinical indicators: Concept of Crohn's disease, epidemiology, classifications, diagnosis, treatment, follow up, and special situations
\end{abstract}

F. Ueno

Ofuna Chuo Hospital, Kanagawa, Japan

T. Matsui

Department of Gastroenterology,

Fukuoka University Chikushi Hospital, Fukuoka, Japan

T. Matsumoto

Division of Lower Gastroenterology,

Department of Internal Medicine, Hyogo College of Medicine,

Nishinomiya, Japan

\section{K. Matsuoka $\cdot$ T. Hibi $(\bowtie)$}

Division of Gastroenterology and Hepatology,

Department of Internal Medicine,

Keio University School of Medicine, 35 Shinano-machi

Shinjuku, Tokyo 160-8582, Japan

e-mail: thibi@z5.keio.jp

M. Watanabe

Department of Gastroenterology and Hepatology,

Tokyo Medical and Dental University, Tokyo, Japan
- Intervention: Diagnosis (interview, physical examination, clinical laboratory tests, imaging, and pathology) and treatment (lifestyle guidance, drug therapy, nutritional therapy, surgery, etc.)

- Outcome assessment: Attenuation of symptoms, induction and maintenance of remission, imaging findings, quality of life (QOL), prevention of complications and harm of therapy

- Methods for developing these guidelines: Described in the text

- Basis of recommendations: Integration of evidence level and consensus of experts

- Cost-benefit analysis: Not implemented

- Evaluation of effectiveness: Yet to be confirmed

- Status of guidelines: Updated version of the first Guidelines published in 2010

- Publication sources: Printed publication available and electronic information in preparation

- Patient information: Not available

- Date of publication: October 2011

These guidelines were intended primarily to be used by practitioners in Japan, and the goal of these guidelines is to improve the outcomes of patients with Crohn's disease.

Keywords Crohn's disease - Guidelines - Practice guidelines $\cdot$ Evidence $\cdot$ Consensus

\section{Contributors to the development of the guidelines}

1. Development Committee

Fumiaki Ueno (Chair); Ofuna Chuo Hospital, Takayuki Matsumoto (Vice-chair); Division of Lower Gastroenterology, Department of Internal Medicine, Hyogo College of Medicine, 
Hiroaki Ito; Kinshukai Infusion Clinic,

Nagamu Inoue; Division of Gastroenterology and Hepatology, Department of Internal Medicine, Keio University School of Medicine,

Kiyonori Kobayashi; Department of Gastroenterology, Kitasato University East Hospital,

Kenji Kobayashi; Division of Endoscopy, Ofuna Chuo Hospital,

Akira Sugita; Department of Surgery, Yokohama Municipal Citizen's Hospital,

Yasuo Suzuki; Department of Internal Medicine, Toho University Medical-Center Sakura Hospital, Yoshinori Noguchi; Department of General Internal Medicine, Nagoya Daini Red Cross Hospital,

Toshiaki Watanabe; Department of Surgery, The University of Tokyo School of Medicine

2. Assessment Committee

Toshiyuki Matsui (Chair); Department of Gastroenterology, Fukuoka University Chikushi Hospital, Mamoru Watanabe (Vice-chair); Department of Gastroenterology and Hepatology, Tokyo Medical and Dental University,

Ryosuke Shoda; Departments of Internal Medicine and General Clinical Practice, East Saitama National Hospital; Nobuo Hiwatashi, Iwaki Kyoritsu General Hospital,

Seiji Bito; Division of Clinical Epidemiology, National Hospital Organization Tokyo Medical Center

3. Research Group of Intractable Inflammatory Bowel Disease

Mamoru Watanabe (Principal investigator); Department of Gastroenterology and Hepatology, Tokyo Medical and Dental University

4. The Japanese Society of Gastroenterology

Toshifumi Hibi (Responsible trustee); Division of Gastroenterology and Hepatology, Department of Internal Medicine, Keio University School of Medicine,

Kentaro Sugano (Chair, Supervision Committee); Department of Gastroenterology, Jichi Medical University Hospital,

Takeo Nakayama (Adviser); Department of Health Informatics, Kyoto University,

Naohiko Yamaguchi (Literature searcher); Toho University Media Center

5. Editors of the English Version

Toshifumi Hibi; Division of Gastroenterology and Hepatology, Department of Internal Medicine, Keio University School of Medicine,
Katsuyoshi Matsuoka; Division of Gastroenterology and Hepatology, Department of Internal Medicine, Keio University School of Medicine, Fumiaki Ueno; Ofuna Chuo Hospital

\section{Introduction}

Crohn's disease (CD) is a complicated disorder of unknown etiology and pathogenesis, involving mainly the small intestine and the colon, but possibly the entire gastrointestinal tract, as well as other organs. Owing to the substantial amount of newer knowledge relevant to the care of $\mathrm{CD}$, the diagnostic and therapeutic strategies for CD have significantly changed, and guidelines to provide appropriate clinical indicators have become necessary. The Japanese Society of Gastroenterology, in collaboration with the Research Group of Intractable Inflammatory Bowel Disease subsidized by the Ministry of Health, Labour and Welfare of Japan, established committees to develop clinical guidelines for CD. The first edition of the guidelines was published in 2010, and an updated version with minor modifications, adding newer therapeutic modalities, was published in 2011. This English version was produced and edited based on the existing updated guidelines.

\section{Purpose and focus}

The purpose of developing these guidelines was to provide appropriate clinical indicators for $\mathrm{CD}$ to contribute to improved outcomes for patients with $\mathrm{CD}$. The statements in these guidelines cover the standard concept of this disease, as well as describing interventions for diagnosis, treatment, and follow up. The guidelines include descriptions of and interventions for gastrointestinal lesions, complications, extra-intestinal complications, and special situations in adults with CD. Special considerations for children or elderly patients are not included.

\section{Users and settings}

The target users of these guidelines are practitioners who provide care for $\mathrm{CD}$ patients in clinical settings. The guidelines are intended not only for the use of gastroenterologists, but also for the use of internists, surgeons, and general practitioners who may have the opportunity to care for patients with CD. Although the present guidelines are not intended for the use of patients, patient information may be developed from the guidelines with minor modifications, as clinical questions are extracted from the patient's point of view.

\section{Features}

The clinical questions (CQs) have been extracted from the patient's point of view. The basic process was carried 
out according to the international standardization of guideline development, with particular emphasis on the existing clinical evidence. When evidence was insufficient or inappropriate for practice in Japan, expert opinions were incorporated. In other words, these guidelines aim to provide clinical indicators, with scientific validity as well as applicability and flexibility. The guidelines were developed by the collaborative efforts of the Japanese Society of Gastroenterology and the Research group of Intractable Bowel Disease subsidized by the Ministry of the Health, Labour and Welfare of Japan.

The major differences from the corresponding existing clinical practice guidelines formulated by the Research Group of the Ministry of Health, Labour and Welfare of Japan are: (1) the present guidelines place emphasis not only on the healthcare provider's point of view but also on the patient's; (2) the statements are based on literature evidence with review by an expert group; (3) each indicator is accompanied by a strength of recommendation, defined according to the level of relevant evidence and the expert consensus; (4) not only specialists but all practitioners were intended as potential users, and (5) the scope and limitations of these guidelines are explicitly presented.

\section{Methods for developing the guidelines}

Independent Committees for developing clinical indicators (Development Committee) and for assessment (Assessment Committee), each of which consisted of experts in gastroenterology, general internal medicine, surgery, and clinical epidemiology, were established according to the methods provided by the Guideline Supervision Committee of the Japanese Society of Gastroenterology. The basic process of development was carried out by repeated correspondence between these two committees by which they prepared, evaluated, and modified statements, eventually creating the most appropriate statements for the guidelines.

The Development Committee extracted CQs in the clinical practice field from the patient's point of view, and an external media center collected literature published up to 2007 relevant to these CQs, mainly through MEDLINE, the Cochrane Library, and the Japan Medical Abstracts Society. In addition, the members of the Development Committee manually added important literature published up to 2008 to the references in their respective areas.

The members of the Development Committee examined the levels of evidence (Table 1) for the literature items. Literature items with an evidence level of III or higher were adopted initially, but those with lower levels of evidence were also adopted if relevant to clinical practice. Original articles, as well as reviews and existing guidelines, were selected, with consideration given to clinical
Table 1 Evidence levels of literature-based information

\begin{tabular}{ll}
\hline I & $\begin{array}{c}\text { Systematic review/Meta-analysis of randomized controlled } \\
\text { trials }\end{array}$ \\
II & Based on one or more randomized controlled trials \\
III & Based on a non-randomized controlled trial \\
IVa & Analytical epidemiological study (cohort study) \\
IVb & Analytical epidemiological study (case-control study or cross- \\
& sectional study) \\
V & $\begin{array}{c}\text { Descriptive study (case report or case series) } \\
\text { VI }\end{array}$ Opinion of an expert committee, or an expert, not based on \\
& patient data \\
\hline
\end{tabular}

interventions that improved patient outcomes, irrespective of the health insurance coverage in Japan. For multiple articles with different levels of evidence, the higher level was adopted. Randomized controlled trials with unwarranted quality were regarded as level III. If a statement was based on an unclear citation, it was considered to be the author's opinion and was regarded as level VI.

Statements of recommendation and the corresponding comments were created on the basis of the selected literature, or, for issues without existing evidence, the statements of recommendation and the corresponding comments were created based on the opinions of the members of the Development Committee. The original statements and comments were evaluated by the Assessment Committee, and repetitive modifications were made by the collaborative work of both committees. The Assessment Committee and three external members evaluated the appropriateness of the statements of recommendation on a 1- to 9 scale (from 1 equaling "most inappropriate" to 9 equaling "most appropriate") according to the Delphi method. The final results after three Delphi rounds were reflected in the adoption of the statements of recommendation and the determination of the grades of recommendation.

As there are some therapeutic modalities that were approved for clinical use after the publication of the Japanese Society of Gastroenterology's Clinical Practice Guidelines for Crohn's Disease (published by Nankodo Co., Ltd., Tokyo, Japan), additional searches of the literature relevant to these therapies were performed, and the same process as that described above was employed to create statements of recommendation for these therapies.

5. Criteria for setting recommendation grades and interpretations

The grade of a recommendation is usually determined parallel to the quality of the evidence on which the statement of recommendation is based, but there are some difficult issues with this process. The level of evidence 
Table 2 Criteria for setting recommendation grades

\begin{tabular}{lllll}
\hline Level of evidence & \multicolumn{4}{l}{ Consensus (median value of Delphi evaluation) } \\
\cline { 2 - 5 } & 8 or more & 7 & $6-4$ & 3 or less \\
\hline I & A & A & C2 & D \\
II & A & B & C2 & D \\
III & B & B & C2 & D \\
IV & B & C1 & C2 & D \\
V & C1 (B*) & C1 & C2 & D \\
VI & C1 $\left(B^{*}\right)$ & C1 & C2 & D \\
\hline
\end{tabular}

Table 3 Interpretations of recommendation grades

A Strong recommendation, with high level of evidence
B Moderate recommendation, with certain level of evidence
Supported by an intermediate level of evidence and considered
to be clinically useful
Supported by a high level of evidence but not considered to be
clinically very useful
Evidence level is low, but usefulness has already been
established in clinical practice

does not assure the quality of the results of clinical studies, but is defined by the study design. There are some timehonored clinical practices without high evidence; on the other hand, there are some novel modalities in the recent literature with a high level of evidence but whose clinical usefulness is yet to be determined. In addition, a clinical intervention directly affecting human life is not a subject for a randomized controlled trial. For these issues, an expert opinion is essential.

We were concerned about the possibility of biased consensus formation owing to the opinions of the dominating expert in the group and the lack of clarity of the impact of the consensus on the recommendations. In these guidelines, the Delphi method was used to form a sound consensus. In addition, the relationship of the consensus to the recommendation grade was clearly defined (Table 2). As a rule, the recommendation grades presented in these guidelines were determined following the above-mentioned principles; however, some statements of recommendation that were considered to be obvious by the consensus of the experts even without good evidence were graded as B rather than $\mathrm{C} 1$ : they are indicated as $\mathrm{B}^{*}$. The meanings of the grades thus determined are described in Table 3. Note that the clinical indicators that were evaluated by the consensus of the experts as not being appropriate (a Delphi median value of 6 or less) were not recommended, as a rule (i.e., those graded as $\mathrm{C} 2$ or D), regardless of the quality of the related literature-based evidence.

\section{Internal review of the guidelines}

The final drafts of the Clinical Practice Guidelines for Crohn's Disease (published by Nankodo Co., Ltd., Tokyo, Japan) were put up on the website of the Japanese Society of Gastroenterology for 2 months to invite opinions from the members of the Society. They were also distributed to a total of 68 research group members and collaborators of the Research Group of Intractable Inflammatory Bowel Disease subsidized by the Ministry of Health, Labour and Welfare of Japan (who represent experts on inflammatory bowel disease in Japan) for their evaluation of the validity of the guidelines. Minor modifications, made by the adoption of some comments, were made by the collaboration of the Assessment and Development Committees.

\section{Applicability of the guidelines}

The literature articles that were used as the basis for creating the statements of recommendation were selected considering their feasibility in clinical practice in Japan. Accordingly, theclinical indicators in these guidelines can be applied to daily clinical practice without any change of current systems/organizations, and therefore they probably do not require any notable additional medical resources.

Examining whether these clinical indicators would be covered by the Japanese health insurance system, it appeared that the great majority of them would fall within the coverage of the insurance system. The exceptional indicators that fall outside the range of insurance coverage are accompanied by a note.

\section{Benefits and risks of applying the guidelines}

The statements of recommendation in these guidelines are standard clinical indicators for $\mathrm{CD}$. They support the decision-making of practitioners, but they neither regulate nor restrict their clinical practice. They are not intended to be a basis for legal judgments. With the support of specialists as needed, and with the flexible use of the guidelines giving sufficient consideration to the patients' values, we believe the guidelines will contribute to the improved quality of clinical practice and patient outcomes.

In actual clinical practice, the patients' values and practitioners' sound judgment are paramount, and it would be inappropriate for these guidelines to be used to control clinical practice, to provide a legal basis for clinical practice, or to restrain practitioners' discretion, and these guidelines would, rather, be considered harmful if used in such manner. 
9. Independence of the guidelines

These guidelines were developed for common objectives by members of the Japanese Society of Gastroenterology and by the Research Group of Intractable Inflammatory Bowel Disease subsidized by the Ministry of Health, Labour and Welfare of Japan. The guidelines were developed independent of any other funding sources and without the cooperation or coordination of any other academic organization, healthcare-providing organization, or patient organization.

A comprehensive list of potential conflicts of interest between health/medical industries and members of the various committees and institutions/organizations who developed the guidelines is provided (see Table 4). Such relationships are related to the individual members or the institutions, and no funding has been provided for the development of these guidelines.

\section{Future issues}

For these guidelines, the statements of recommendation were created with an emphasis on literature-based evidence in response to the CQs raised from the patients' point of view, and the statements were reviewed and modified by a group of experts. The recommendation grades were determined on the basis of the levels of evidence and then clearly integrated by formal consensus. As a result, we believe that these guidelines would possess internal validity, and clinical applicability and flexibility. They have not, however, been evaluated for their effectiveness, in terms of actual contribution to the improved quality of clinical practice for $\mathrm{CD}$, and this is an issue to be addressed later.

With the accumulation of newer evidence, a process of revision of the guidelines will be necessary. These guidelines may need to be revised and supplemented by 3 years or so after their publication. Assessment by the users should be considered for revision, and therefore constructive criticisms are invited.

\section{Disclosure of conflicts of interest}

1. Disclosure of potential conflicts of interest in relation to health/medical industries

The health/medical industry entities from which members of the committees for developing the guidelines were provided with remuneration according to medical professional contracts, or for lectures, written contributions, supervision of publications, etc., or from which they have received research funding, are listed in Table 4 on their own declaration, regardless of their relationships with the makers of products used for CD. The names of the health/ medical industry entities are those as of June 2011. Publishers and non-profit organizations that adopt a position of neutrality are excluded.

2. Means of minimizing conflicts of interest.

The statements of recommendation were created on the basis of literature-based evidence. The consensus of the Assessment Committee was formally generated according to the Delphi method to avoid the domination of any particular members. The recommendation grades were determined on the basis of the levels of evidence with integration by formally developed consensus, as clearly indicated by the median values of Delphi rounds. In general, these guidelines were developed in accordance with the proposed Conference on Guideline Standardization (COGS) standard [1].

Table 4 Disclosure of conflicts of interest

\footnotetext{
Medical professional contracts with the following companies:

Ajinomoto, Astellas, Abbott, Eisai, LTT Bio-Pharma, Otsuka, Kyorin, Zeria, Mitsubishi Tanabe, Chugai, FUJIFILM Medical, Bristol, Merck Serono

Remuneration for lectures, written contributions, supervision of publications, etc., from the following companies:

Asahi Kasei Kuraray Medical, Ajinomoto, ASKA, Astellas, AstraZeneca, Abott, EN Otsuka, Eisai, MSD, Otsuka, Otsuka Pharmaceutical Factory, Olympus Medical Systems, KAKEN, Kyorin, Kyowa Hakko Kirin, GSK, Shionogi, JIMRO, Zeria, DAIICHI SANKYO, Tyco, Dainippon Sumitomo, Taiho, Takeda, Mitsubishi Tanabe, Chugai, TSUMURA, Terumo, TORAY, Torii, NIPPON KAYAKU, Nihon Schering, Novartis, FUJIFILM Medical, Bristol, Boston Scientific Japan, Merck Serono, Yakult, Janssen, UCB

Provision of research funding from the following companies:

Asahi Kasei Kuraray Medical, Ajinomoto, Astellas, AstraZeneca, EN Otsuka, Eisai, MSD, Otsuka, Otsuka Pharmaceutical Factory, Olympus Medical Systems, KAKEN, Kyowa Hakko Kirin, Kyorin, KUREHA, GSK, Shionogi, JIMRO, J\&J, Zeria, DAIICHI SANKYO, Tyco, Taisho Toyama, Dainippon Sumitomo, Taiho, Takeda, Mitsubishi Tanabe, Chugai, TSUMURA, Torii, Pfizer, FUJIFILM Medical, Bristol, MIYARISAN, Merck Serono, Yakult, UCB

Company with potential conflicts of interest though family members:

Shionogi
} 


\section{How to read the guidelines}

In the text, CQs are presented according to clinical categories, and each CQ is followed by one or more corresponding statements of recommendation. Each of the statements is accompanied by a recommendation grade that indicates the strength of the recommendation. The recommendation grade, which is defined in Table 3, is followed by the levels of evidence of the literature articles that are the basis for the recommendation (in Japan and overseas) and the value of the expert group consensus (i.e., the Delphi evaluation median value). The comments provide a general description of the clinical practice related to each $\mathrm{CQ}$. The lists of references are presented after the text.

\section{Clinical questions (CQs) and statements}

\section{Disease concept}

\section{I-1. Definition}

CQ1: What is CD? [2]

- $\mathrm{CD}$ is a chronic disease of unknown causes that mainly presents as granulomatous inflammatory lesions of the gastrointestinal tract. C1 (Japan VI, overseas VI; 8).

\section{Comments}

$\mathrm{CD}$ is a chronic inflammatory disease of the gastrointestinal tract characterized by discretely distributed transmural granulomatous inflammations and fistulas. Lesions of CD may occur throughout the entire gastrointestinal tract, but they occur most commonly in the small intestine, the colon (especially the ileocecal region), and the perianal region [2]. The disease occurs at a young age, and lasts chronically, with remissions and relapses of symptoms and signs such as abdominal pain, diarrhea, hematochezia, fever, perianal symptoms, and weight loss, resulting in a reduced quality of life for the patients. Also, CD may cause extra-intestinal complications in the joints, the skin, the eyes, and other parts of the body. CD and ulcerative colitis (UC) are together referred to generically as inflammatory bowel disease (IBD). Although they have common and/or similar features, they are considered to be mutually distinct.

\section{I-2. Epidemiology}

CQ2: How common is CD, in what age group does it occur, and in Japan, are there any differences from other countries? [2-7]

- The number of CD patients in Japan is steadily increasing, with a current estimate of more than 30,000 .
$\mathrm{CD}$ is more prevalent among men than women, at an approximate ratio of 1.8: 1.0. B* (Japan V; 9).

- CD occurs at comparatively young ages, more commonly from the late teens to the early $30 \mathrm{~s}$. B* (Japan $\mathrm{V}$; 9)

- The prevalence and incidence of CD in Europe and North America are higher than those in Japan, and female preponderance in those areas is noted. C1 (overseas $\mathrm{V} ; 8$ ).

\section{Comments}

A Japanese nationwide epidemiological survey in 1991 reported that the prevalence of $C D$ was 5.85 per 100,000 (7.94 among men and 3.83 among women) and that the incidence of CD was 0.51 per $100,000(0.71$ among men and 0.32 among women) [3]. The figures were clearly lower than those in Europe and North America. Although no such survey has been conducted since then, the estimated number of $\mathrm{CD}$ patients has steadily increased in Japan [4], and more than 30,000 patients received registered medical services for $C D$ in 2009.

The onset of CD is usually in young people, commonly in the early third decade among men and in the late second decade among women [2-4]. According to the Japanese national registration record for medical services, it is estimated that $\mathrm{CD}$ occurs more commonly in the third and early fourth decades among men and in the late second decade among women [5].

The incidence in countries overseas is usually higher than that in Japan, although it varies from region to region; there are a substantial number of regions with a CD incidence rate of around 10 per 100,000 in Europe and North America. It has been shown that the prevalence of this disease has been increasing globally from year to year [6]. In general, $\mathrm{CD}$ is more common in women in Europe and North America, unlike in Japan [7]. At present, Japan is ranked in the middle for the prevalence and incidence rates of CD, together with South Korea, Oceanic countries, and South Africa [4].

\section{I-3. Etiology}

CQ3: What causes CD? Is it inherited? What are the risk factors? [8-19]

- The causes of CD have not been identified. C1 (Japan VI, overseas VI; 8).

- A tendency for familial occurrence is noted. B (overseas IVa; 8). 
- Some causal relationships between diet and CD have been reported, though the evidence is not conclusive. B (Japan IVb, overseas IVb; 8).

- Smoking is a risk factor for CD. B (overseas III; 8).

- Nonsteroidal anti-inflammatory drugs (NSAIDs) and oral contraceptives are potential factors for the exacerbation of CD. C1 (overseas IVb; 7).

\section{Comments}

The causes of CD have not yet been identified. The current international consensus is that the intestinal inflammation is caused by a disordered immunomodulatory mechanism, with exposure to various environmental factors in an individual with genetic susceptibility [8-10]. A somewhat higher incidence of $\mathrm{CD}$ is reported among relatives [11, 12], and reports of familial clustering indicate the presence of a genetic mechanism. Studies are underway to find the disease susceptibility genes for CD.

Regional differences in the incidence of $\mathrm{CD}$ suggest a causal relationship between diet and $\mathrm{CD}$, and many clinical and epidemiological studies have been reported. Some overseas reports found a causal relationship between CD and high intakes of carbohydrates (particularly sugar) [13, 14], and some reports from Japan showed a causal relationship between CD and "fast foods" with high contents of fats and sugar [15]. However, no food has been concluded to be a risk factor for CD.

Smoking is considered to be a risk factor for CD. Reports have shown that smoking is not only related to the onset, relapse, and exacerbation of $\mathrm{CD}$, but also that smoking cessation lowered the postoperative recurrence rate [16]. Smoking also affects the efficacy of infliximab treatment [17].

Among many drugs evaluated, NSAIDs and oral contraceptives have been shown to be associated with the onset and exacerbation of CD [18, 19].

\section{I-4. Pathophysiology, classification, and disease activity}

CQ4: What kind of pathophysiological conditions are present in $\mathrm{CD}$, and how are they recognized? [20]

- To provide appropriate treatment, it is necessary to exactly recognize the disease extent, disease pattern, and degree of disease activity. B* (Japan VI, overseas: VI 9).

\section{Comments}

The pathophysiological conditions of $\mathrm{CD}$ are complex, but correct recognition is the first step for appropriate management. It is important to identify the distribution of the lesions, to recognize the disease pattern, and to assess disease activity/severity.

The lesions are often found in the small intestine, the colon (particularly in the ileocecal region), and the perianal region, and are classified as the ileal, colonic, and ileocolonic types. Note, however, that lesions can occur at any site in the gastrointestinal tract; moreover, CD may have extra-intestinal complications causing systemic involvement. Treatment plans differ according to the sites of involvement.

According to an international proposal, the disease patterns can be classified as "Inflammatory", "Penetrating", and "Stricturing" [20]. Recognition of the disease pattern is also important for appropriate treatment.

Furthermore, it is necessary to assess the disease activity. Treatment during the remission phase when the symptoms have abated or are absent is different from treatment during the active phase when various symptoms interfere with daily life. For the objective evaluation of the disease activity and severity, the Crohn's Disease Activity Index (CDAI) is available, but it is not suitable for daily clinical practice. The Index of Inflammatory Bowel Disease (IOIBD) is simple, but it does not assist in the selection of appropriate treatment options. At present, there is no universal classification of $C D$ severity available for use in Japan. In general clinical practice, the disease activity can be assessed by the comprehensive evaluation of subjective symptoms, clinical findings, and laboratory investigations.

\section{I-5. CD progression}

CQ5: How does CD progress in the long term? Is there an increased risk of cancer? Is life expectancy shortened? [21-29]

- CD persists, with remissions and relapses, for a long period of time. C1 (Japan VI, overseas VI; 9).

- During disease progression, the daily life of $\mathrm{CD}$ patients is often disturbed. C1 (Japan V, overseas V; 8).

- The incidence of cancer among CD patients is slightly elevated. B (Japan V, overseas IVb; 8).

- The life expectancy of CD patients is slightly shorter than that of healthy individuals. C1 (Japan V, overseas IVb; 7).

\section{Comments}

$\mathrm{CD}$ is a disease that persists for a long period of time, with repeated active phases and remission phases. The symptoms and complications in the active phase make it difficult for patients to live a normal daily life. In countries 
overseas, $15 \%$ of $\mathrm{CD}$ patients become unable to work 5-10 years after the diagnosis [21]. According to a crosssectional study in Japan, fewer than $30 \%$ of CD patients are able to work in full-time employment throughout the year [22]. Intra- and extra-intestinal complications are considered to have the most negative impact on the activities of daily living (ADL) among CD patients [23].

An overseas meta-analysis report indicates that the relative risks of cancer in the colon and the small intestine in CD patients are 2.4 and 28.4, respectively [24]. Although the relative risk of small-intestinal cancer is conspicuously higher than that of the colon, its significance is not clear because the absolute number of these cancer cases is very small. According to a Japanese report on the association of $\mathrm{CD}$ and cancer in the colon, the small intestine, and the anal canal, most cases of such cancers are discovered at an advanced stage. Most reports on the incidence of cancer in CD patients in Japan are based on case-series studies, but some controlled studies suggest that the incidence in Japan is similar to that in Europe and North America [25, 26].

Six of seven overseas area cohort studies estimate that the mortality in CD patients has been greater than 1.0, and has been stable in the past 40 years [27]. Two reports from Japan showed different conclusions: one reported that mortality among CD patients was higher, while the other reported that mortality was similar to that of the healthy population $[28,29]$. In general, $C D$ does not seem to significantly reduce the life expectancy of the patients.

\section{Diagnosis (Fig. 1)}

\section{II-1. Clinical symptoms}

CQ1: What are the clinical symptoms of CD? [30]

- Abdominal pain and diarrhea are the most common symptoms. Symptoms due to perianal lesions and hematochezia are also commonly encountered. C1 (Japan VI, overseas V; 8).

- Although systemic symptoms and signs such as weight loss, fever, general malaise and anorexia, and oral aphthous ulcerations are often observed, they are not highly specific for CD. C1 (Japan VI, overseas V; 8).

\section{Comments}

Abdominal pain $(70 \%)$ and diarrhea $(80 \%)$ are commonly encountered at the time of diagnosis. Hematochezia is observed in $30 \%$ of patients, but is usually not massive. Generally, abdominal pain is more common in the smallintestinal type, whereas hematochezia and diarrhea are more common in the colonic type. In the course of the disease progress, perianal lesions are observed in more than $50 \%$ of patients, and fistulas and abscesses manifest in approximately $15 \%$ of patients [30].

Systemic symptoms and signs such as weight loss and fever are found in $40-70 \%$ of patients at the time of diagnosis. Weight loss is more common in the small-intestinal type. Systemic symptoms such as general malaise and anorexia, aphthous stomatitis, and shallow ulcers in the oral cavity are often observed during the progress of the disease, but they are not highly specific for $\mathrm{CD}[30]$. Extra-intestinal complications such as lesions in the joints, skin, and eyes are observed in approximately $2-10 \%$ of patients [30].

CQ2: What are the complications associated with CD? [30-35]

- Intestinal complications of CD include stenosis, fistulas (internal and external), abscess formation, massive hemorrhage, and colorectal cancer. B* (Japan V, overseas V; 9).

- Extra-intestinal complications of $\mathrm{CD}$ include joint lesions (e.g., joint pain, acute peripheral arthritis, and reactive arthritis), skin lesions (e.g., erythema nodosum, Sweet's disease, and pyoderma gangrenosum), eye lesions (e.g., iritis and episcleritis), and primary sclerosing cholangitis (PSC). C1 (Japan V, overseas V; 8).

- Complications to which children are susceptible include growth retardation, osteoporosis, and angitis. C1 (Japan V, overseas V; 7).

\section{Comments}

Intestinal complications of $\mathrm{CD}$ include stenosis, internal and/or external fistulas, and abscess formation, any of which are not infrequently candidates for surgical interventions. Complications increase with the disease progress [31]. CD with intestinal complications is known as the disabling type [31], and thus it is important to prevent these complications or treat them appropriately in order to maintain the patients' QOL. Massive hemorrhage occurs in $0.6-5 \%$ of patients, and it usually arises from anastomotic sites and the small intestine [30].

Signs of joint lesions include joint pain or acute peripheral arthritis (type 1: fewer than five joints, mainly in the large joints, associated with the $\mathrm{CD}$ activity) and reactive arthritis (type 2: polyarthritis in the small joints, not associated with the CD activity) [32]. Joint symptoms are found in $30 \%$ or more (arthralgia, $14.3 \%$; type 1 arthritis, $6 \%$; type 2 arthritis, $4 \%$; and axial arthropathy, $9.9 \%$ ) [32]. The reported association of $\mathrm{CD}$ with skin lesions, including erythema nodosum, Sweet's disease, and pyoderma gangrenosum, has been increasing. According to 
Fig. 1 Diagnostic approach in Crohn's disease. CRP

$\mathrm{C}$-reactive protein

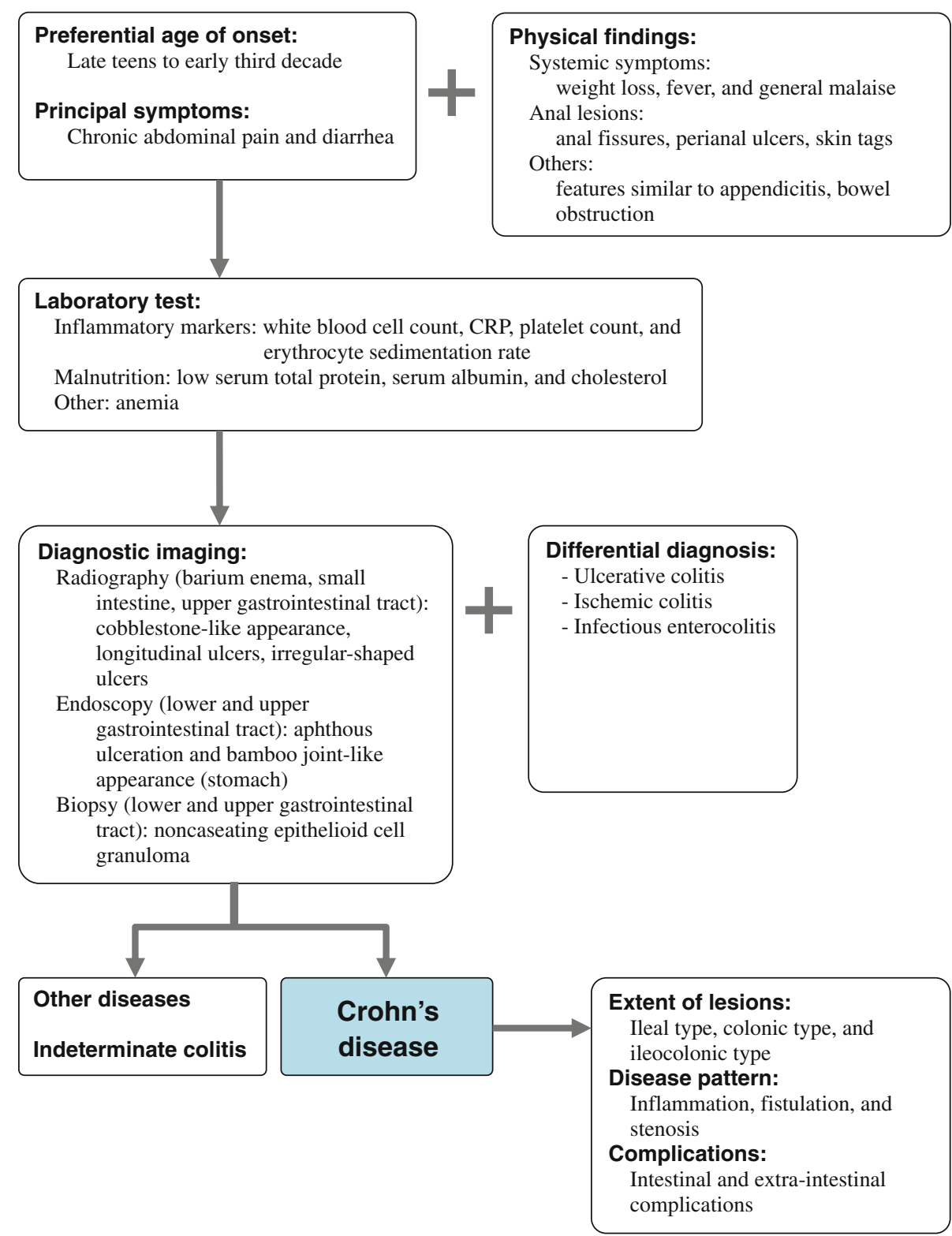

an overseas report, approximately $2.2 \%$ of IBD patients have skin symptoms $[33,34]$. The frequency of erythema nodosum is about three times as high as that of pyoderma in IBD patients. Both skin conditions are more likely to manifest as a complication of CD rather than UC. Sweet's disease is rarely found in CD patients (30 reported cases in Europe and North America) [35]. Nonspecific skin eruptions are more often seen among CD patients than in healthy people, but they are not correlated with the disease activity.

Iritis and episcleritis are found in 1-2\% of IBD patients [34]. PSC as a complication of CD is found at a rate of $1-3 \%$, which is lower than that in UC. Psoriasis is more common in $\mathrm{CD}$ patients and their siblings than in the general population.
Complications more often found in children include growth retardation, osteoporosis, and angitis [30]. The frequency of extra-intestinal complications is higher among children with IBD than in adults with IBD, and some reports indicate that the rate of such complications in children is as high as $35 \%[30,32,34]$.

CQ3: What kind of perianal lesions are caused by CD? [36-38]

- Perianal lesions include anal fissures, anal ulcers, skin tags, anal fistulas, perianal abscesses, anovaginal fistulas, cavitating ulcers, piles, and anal canal cancer. C1 (Japan V, overseas V; 8). 


\section{Comments}

Perianal lesions are found in more than $50 \%$ of patients with $\mathrm{CD}$, and often precede other symptoms (36-81\%). The frequency of perianal lesions as complications is significantly higher in cases of CD with rectal stenosis than in those without such stenosis [36-38].

Hughes [37] classified perianal lesions according to the pathological conditions, as follows: primary lesions, i.e., deep ulcers (deep anal fissures and anal ulcers) caused by CD itself; secondary lesions, i.e., secondary lesions originating from the primary lesions via infection or other causes; and incidental lesions, i.e., lesions not related to CD. Recently, cases of anal canal cancer as a complication have also been reported in Japan. It is necessary to survey these lesions carefully in patients with long-term progression of the disease.

\section{II-2. Medical interview and physical examination}

CQ4: What kind of symptoms and physical findings make CD suspected? [39]

- Chronic abdominal pain and/or diarrhea in young individuals suggest the possibility of CD, especially when accompanied by weight loss and fever. C1 (Japan VI, overseas VI; 8).

- On physical examination, characteristic perianal lesions (preferably checked by colorectal surgeons familiar with CD), findings similar to appendicitis, bowel obstruction, and rectal bleeding indicate CD. C1 (Japan VI, overseas VI; 7).

- Although the onset of CD is usually at young ages, it is not rare in the elderly. C1 (Japan VI, overseas VI; 7).

\section{Comments}

According to the Proposed Diagnostic Criteria for Crohn's Disease [39], this disease presents with initial symptoms of "abdominal pain, diarrhea, weight loss, fever, perianal lesions, symptoms particularly similar to appendicitis, bowel obstruction, intestinal perforation, and/or massive hemorrhage. Furthermore, it may occur with perianal lesions and/or fever (of unknown causes), without abdominal symptoms".

\section{II-3. Diagnostic strategies}

CQ5: If CD is suspected, how do physicians proceed to the diagnosis? What kind of investigations are required? $[40,41]$

- Obtain blood tests to check for inflammatory activity, malnutrition, and iron-deficiency anemia. C1 (Japan VI, overseas VI; 8).
- Use imaging procedures to check for morphological findings characteristic of CD. C1 (Japan VI, overseas VI; 8).

- Exclude infectious enterocolitis (including tuberculosis), if necessary, by stool culture and other tests. C1 (Japan VI, overseas VI; 8).

- Apply tests to check for intestinal complications according to the symptoms. C1 (Japan VI, overseas VI; 8).

\section{Comments}

Apply blood tests to examine for abnormal inflammatory responses (in terms of white blood cell count, C-reactive protein [CRP], platelet count, and erythrocyte sedimentation rate $[\mathrm{ESR}]$ ), malnutrition (low serum total protein and albumin, and/or low total cholesterol), and anemia.

Employ lower gastrointestinal endoscopy (including histological evaluation), barium enema, and/or smallintestinal contrast radiography as imaging examinations. Check for longitudinal ulcers, a cobblestone-like appearance, stenosis, and fistulas, which are characteristic of CD in such examinations. Use upper gastrointestinal endoscopy with biopsy as much as possible to detect multiple aphthous ulcerations, ulcers, stenosis, and a cobblestonelike appearance as lesions of $\mathrm{CD}$ in the upper gastrointestinal tract [40, 41]. Imaging procedures are usually used to exclude similar disorders. Stool cultures and serum antibodies are utilized to check for infectious enterocolitis.

Regarding intestinal complications, use computed tomography (CT) and/or magnetic resonance imaging (MRI) to check for the presence and the severity of perianal abscesses, anal fistulas, and intra-abdominal abscesses.

CQ6: What morphological examinations are necessary to make a diagnosis of $\mathrm{CD}$ ? $[40,41]$

- Lower-gastrointestinal endoscopy, barium enema radiography, small-intestinal radiography, upper-gastrointestinal endoscopy, upper-gastrointestinal contrast radiography, and histopathological examination are necessary. B* (Japan VI, overseas VI; 9).

\section{Comments}

The common sites of involvement by $\mathrm{CD}$ are the colon and the distal ileum; therefore, barium enema radiography, lower-gastrointestinal endoscopy (including examination of the terminal ileum and histological evaluation of biopsy specimens), and small-intestinal radiography are usually conducted prior to other examinations. 
Although upper-gastrointestinal endoscopy is not indispensable, it should be used in cases where a definitive diagnosis could not be made by barium enema radiography or lower-gastrointestinal endoscopy.

Small-intestinal endoscopy is helpful in cases where CD lesions are not found on the lower and upper gastrointestinal endoscopic examinations or on contrast radiography despite the clinical suspicion of $\mathrm{CD}$. Although capsule endoscopy is used overseas to detect lesions in the small intestine, it has not been approved for clinical use in Japan because of potential complications at the site of a stenosis.

CQ7: What kind of laboratory markers are useful to evaluate the activity of CD? [42-44]

- Markers of inflammatory response (CRP and ESR) are considered to correlate with the disease activity. C1 (Japan VI, overseas VI; 8).

- Nutritional indices (serum total protein and serum albumin) also reflect the disease activity in many cases. C1 (Japan VI, overseas VI; 7).

- There is no single index with which to quantitate disease activity to enable an objective assessment; therefore, it is necessary to assess CD disease activity in a comprehensive manner. C1 (Japan VI, overseas VI; 9).

\section{Comments}

The disease activity of CD is generally assessed by the CDAI or IOIBD. The CDAI is the most commonly used assessment tool worldwide [42]. CD causes lesions at any site along the entire gastrointestinal tract, and the sites and extent of lesions are related to the disease activity. Generally, the markers of inflammatory response (CRP and ESR) are comparatively well correlated with the CDAI scores indicating the disease activity, but with occasional discrepancies. In patients with severe and extensive disease, especially those with extensive small-intestinal lesions, hypoproteinemia is often present. However, values for nutritional indices may be affected by treatments such as nutritional therapy. Accordingly, no single laboratory test can contribute to the assessment of the disease activity.

The Endoscopic Index of Severity of Crohn's Disease (CDEIS) has been proposed as an endoscopic index of disease activity $[43,44]$. This index is obtained by dividing the intestinal tract into five segments (the rectum, the sigmoid/descending colon, the transverse colon, the ascending colon/cecum, and the ileum), determining scores and obtaining subtotals according to the depth and length of ulceration and the area of lesions in each segment, and then calculating an average based on the number of segments with lesions, and adding points for stenosis to the average. Calculation of values in this index is complicated and timeconsuming. The index is not commonly used to evaluate disease activity in clinical practice.

\section{II-4. Endoscopy}

CQ8: When is endoscopic examination necessary to make a diagnosis of CD? [39, 40, 43-46]

- When clinical symptoms and the laboratory test results suggest $\mathrm{CD}$, promptly examine the patient using lowergastrointestinal endoscopy (including an observation of the terminal ileum) and histological evaluation of biopsy specimens. C1 (Japan VI, overseas VI; 8).

- Examine the patient using upper-gastrointestinal endoscopy when a definitive diagnosis has not been made with lower-gastrointestinal endoscopy or when the patient complains of symptoms in the upper gastrointestinal tract. C1 (Japan VI, overseas VI; 8).

\section{Comments}

CD can affect the entire gastrointestinal tract, but the common sites are the colon and the distal ileum. When clinical symptoms and the laboratory test results suggest $\mathrm{CD}$, promptly examine the patient using lower-gastrointestinal endoscopy, including an observation of the terminal ileum, to make a definitive diagnosis, to determine the extent and severity of inflammation, and also to take a biopsy sample for histological examination [40, 43-46]. Endoscopy has recently been employed for the treatment of stenosis. Small-intestinal balloon endoscopy may be useful.

Upper-gastrointestinal lesions are not rare and may occur at a high rate (17-75\%) in patients with $\mathrm{CD}$, with or without related symptoms. The Japanese proposed diagnostic criteria for Crohn's disease [39] refer to irregularshaped ulcers and aphthous ulcerations found in both the upper and lower gastrointestinal tract as minor findings39. Accordingly, to make a definitive or differential diagnosis of $\mathrm{CD}$, it is useful to explore lesions by using upper-gastrointestinal endoscopy and by examining biopsy samples for histological evaluation (to check for the presence of noncaseating epithelioid cell granuloma).

CQ9: What endoscopic findings are characteristic of CD? [46, 47]

- Lower-gastrointestinal endoscopic findings characteristic of CD include discrete or segmental lesions (socalled skip lesions), a cobblestone-like appearance, 
longitudinal ulcers, irregular-shaped ulcers, multiple aphthous ulcerations, abnormal narrowing and/or stenosis, and fistulas (internal and/or external fistulas). B* (Japan V, overseas VI; 9).

- Upper-gastrointestinal endoscopic findings characteristic of CD include a bamboo joint-like appearance, a notch-shaped appearance, cobblestone-like appearance, multiple aphthous ulcerations, erosion, irregular-shaped ulcers, bead-like protrusions, nodular folds, granular mucous membrane, and stenosis. C1 (Japan V, overseas VI; 8).

\section{Comments}

It was reported that lower-gastrointestinal endoscopic findings make it possible to differentiate $89 \%$ of CD cases from UC among suspected cases of IBD [46]. Findings helpful in differentiating CD from UC are discrete lesions, cobblestone-like appearance, aphthous ulcerations and longitudinal ulcers, and perianal lesions [47].

Frequently encountered upper-gastrointestinal lesions of $\mathrm{CD}$ are a bamboo joint-like appearance in the stomach, gastric and duodenal erosions and/or ulcers, and duodenal notch-like protrusions and/or longitudinal erosions.

CQ10: Is examination of the entire gastrointestinal tract necessary in the diagnosis of CD? [40, 41, 47]

- Examinations of the lower gastrointestinal tract (using endoscopy or barium enema radiography) are almost indispensable for making a diagnosis of CD. B* (Japan VI, overseas VI; 9).

- Even after a definitive diagnosis has been made, it is preferable to examine the patient using small-intestinal contrast radiography and upper-gastrointestinal endoscopy. C1 (Japan VI, overseas VI; 8).

\section{Comments}

For the definitive diagnosis of $\mathrm{CD}$, use lower-gastrointestinal endoscopy with biopsy for histopathological evaluation before undertaking other investigations. Even after the diagnosis is made, it is preferable to investigate the entire gastrointestinal tract, using small-intestinal contrast radiography and upper-gastrointestinal endoscopy, in order to determine the disease type, as determination of the type will contribute to the selection of the appropriate treatment and scheduling of the follow up. In cases where the results of a lower-gastrointestinal endoscopic examination do not produce a definitive diagnosis, it is absolutely necessary to explore lesions in the small intestine and the upper gastrointestinal tract $[40,41,47]$.
If lesions are not found using small-intestinal contrast radiography or upper- and lower-gastrointestinal endoscopy despite clinical suspicions of $\mathrm{CD}$, exploration of the small-intestinal lesions using capsule endoscopy may be useful in some cases; however, this procedure is not yet approved for clinical use in Japan for suspected cases of CD. The usefulness of small-intestinal endoscopy in the diagnosis of CD has not been established clearly, although it is useful in some cases of diagnostic difficulty.

\section{II-5. Contrast radiography}

CQ11: When is contrast radiography necessary to make a diagnosis of CD? [47-49]

- As CD may be complicated with intestinal stenosis, fistulas, abscess, and/or adhesions, the addition of barium enema radiography to colonoscopy would be advisable. C1 (Japan VI, overseas VI; 8).

- Even if a diagnosis of CD is made by barium enema radiography, small-intestinal contrast radiography is valuable to determine the extent of the lesions and to establish treatment strategies. C1 (Japan VI, overseas VI; 8).

\section{Comments}

Barium enema radiography is helpful to overview the entire colon and rectum, and small-intestinal contrast radiography can be performed safely if there is no severe stenosis in the colon. To explore small-intestinal lesions, contrast radiography is still useful, with a sensitivity of 85-95\% and specificity of 89-94\% to detect typical lesions of CD [47-49].

CQ12: Which findings of contrast radiography are characteristic of CD? [49-52]

- Longitudinal ulcers (asymmetric sclerotic appearance), cobblestone-like appearance, stenosis, aphthous ulcerations, irregular-shaped ulcers, fissures, and fistulas are typically found. B* (Japan V, overseas VI; 9).

\section{Comments}

A longitudinal ulcer is an ulcer $5 \mathrm{~cm}$ or longer that runs along the longitudinal direction of the gastrointestinal tract on the mesentery side in the small intestine and along the teniae coli in the colon, varying in width from a wide band to a thin line. Shortening of the mesentery side of the small intestine due to longitudinal ulcers produces an asymmetric 
sclerotic appearance, which is found in approximately $84 \%$ of CD cases [49-52].

A cobblestone-like appearance is where scattered polyplike protrusions are formed in an area of mucous membrane surrounded by a longitudinal ulcer and with smaller ulcers running transversely. It is assumed that mucosal edema, shortening of mucosal muscle, inflammatory cell infiltration, and fibrosis produce such a distinctive feature [52].

\section{II-6. Other imaging procedures}

CQ13: How can imaging procedures such as CT and abdominal ultrasonography (US) contribute to making a diagnosis of $\mathrm{CD}$ ? [53]

- CT and US are useful for evaluating the extent and severity of gastrointestinal inflammation, and for the detection of abscess formation. C1 (Japan VI, overseas VI; 8).

\section{Comments}

CT and US can be used to assess intestinal inflammation, based on thickening of the intestinal walls and the increased density of surrounding fatty tissues. Contrast CT and MRI are helpful in detecting abscess formation. CT colonography, although useful in assessing lesions proximal to a stenosis, is not universally available [53].

\section{II-7. Histopathological examination}

CQ14: What pathological findings are characteristic of $\mathrm{CD}$ ? [52, 54]

- Findings for a definitive diagnosis of CD include: (1) noncaseating epithelioid cell granuloma, (2) transmural inflammation, (3) fissure, and (4) ulcers. C1 (Japan VI, overseas $\mathrm{VI} ; 8$ ).

\section{Comments}

Abnormal alignment of crypts and basal cell plasmacytosis are found in the biopsy specimen, and these are findings in common with those of IBD. A key to differentiating CD from UC is focal inflammation. Granuloma is composed of such cells as epithelioid cells, macrophages, lymphocytes, and multinucleate giant cells. Although noncaseating epithelioid cell granuloma is a principal basis for a diagnosis of CD, it is detected in 40-60\% of surgical specimens, and in only 15-36\% of biopsy specimens [54]. Multiple biopsy specimens for serial sections may improve the rate of detection of granuloma. Note, however, that multinucleate giant cells may be found in foreign-body granulomas, and that noncaseating epithelioid cell granulomas may be found in tuberculosis [52].

In transmural inflammation, focal aggregations, mainly of lymphocytes, are found to be transmurally distributed unevenly. Lymphangiectasis, edema, and fibrosis are also found. Disproportionate inflammation, manifested more strongly in the submucosa than in the lamina propria, is a convincing key to the biopsy diagnosis of CD. Fissure formation is a vertical tissue defect along a lymphatic duct.

\section{II-8. Definitive diagnosis}

CQ15: How can a definitive diagnosis be made? What diagnostic criteria are used? [39]

- If CD is suspected based on medical interview, physical examination, and laboratory test results, gastrointestinal investigations should be conducted. C1 (Japan VI, overseas VI; 9).

- The Japanese proposed diagnostic criteria for Crohn's disease [39] (Table 5) consist mainly of morphological findings of the gastrointestinal tract. C1 (Japan VI; 8).

\section{Comments}

Among major findings, longitudinal ulcers in CD can be differentiated from those in UC or ischemic colitis by the presence of protrusions due to inflammatory edema. The cobblestonelike appearance in $\mathrm{CD}$ involves dense protrusions of mucous membrane of uneven sizes, large or small, surrounded by a longitudinal ulcer and smaller ulcers. This appearance may be seen in ischemic colitis, but in ischemic colitis the protrusions are less dense and hyperemia is more intense.

CQ16: If a diagnosis of $\mathrm{CD}$ is not definitive, what should be done? [54, 55]

- In indeterminate colitis where it is difficult to differentiate $\mathrm{CD}$ from $\mathrm{UC}$, choose treatment strategies for the more suspected disease, observe progress with regular check-ups, and make a definitive diagnosis as soon as the features of one or the other disorder become dominant. C1 (Japan VI, overseas VI; 8).

- If the diagnosis of CD is not definitive, as in patients with only aphthous ulcerations, observe progress with regular check-ups, and a definitive diagnosis of $\mathrm{CD}$ can be made when morphological examinations fulfill the criteria for the diagnosis. C1 (Japan VI, overseas VI; 8). 
Table 5 Proposed diagnostic criteria for Crohn's disease in Japan (as revised in February 2011) [39]

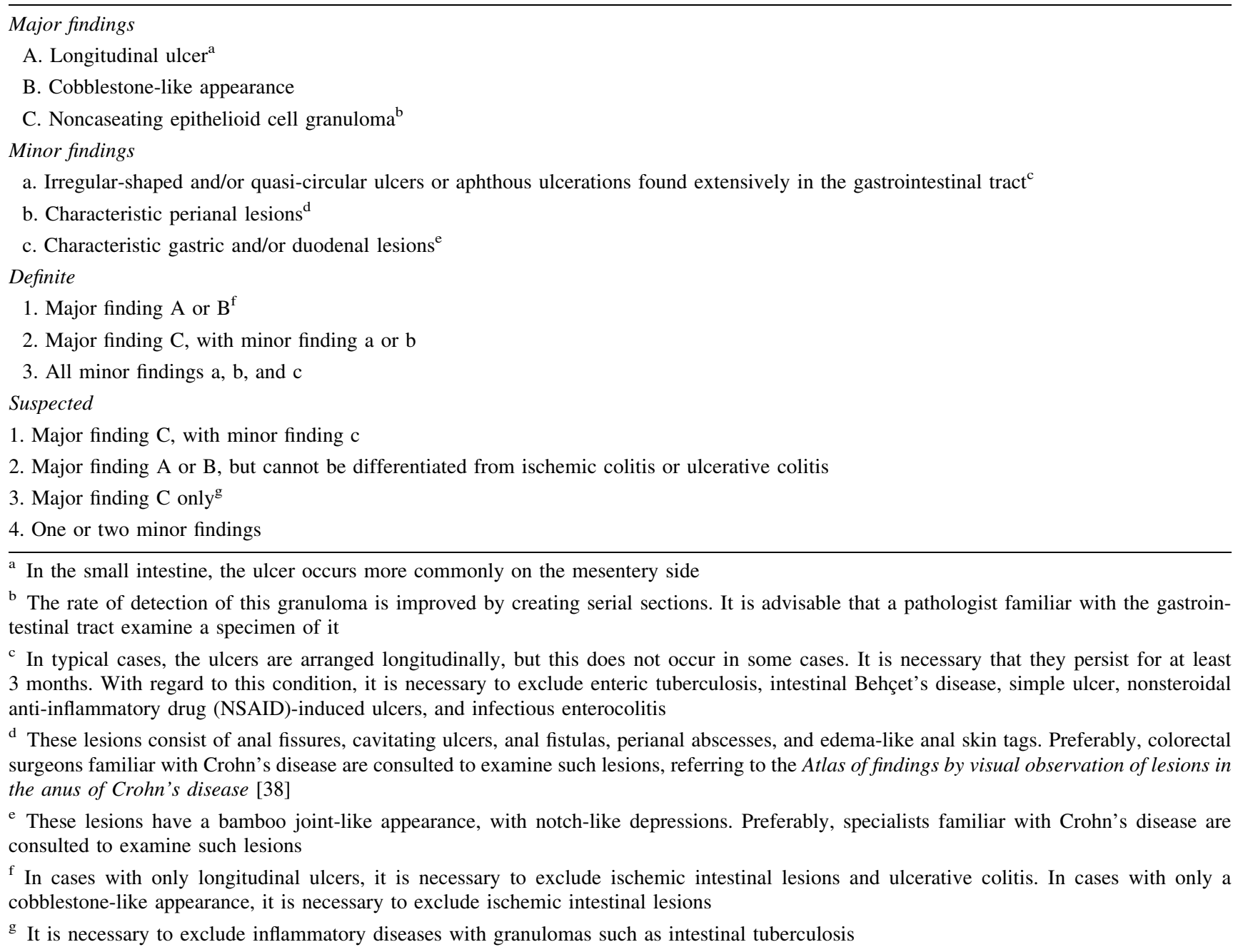

\section{Comments}

The number of reports of indeterminate colitis (IC), which has clinical and histopathological features of both UC and $\mathrm{CD}$ and is thus difficult to differentiate from either of these conditions, is increasing [54, 55]. IC accounts for approximately $4 \%$ of IBD cases in Japan [55]. Even after intestinal resection, in approximately $5 \%$ (range 1-20\%) of cases, a definitive diagnosis cannot be made, because of overlapping histopathological features [54, 55]. Although the usefulness of serum anti-Saccharomyces cerevisiae (ASCA) and anti-neutrophil cytoplasmic antibody (ANCA) measurements has been reported, their diagnostic accuracy for IC has not been established. At present, serial observations with endoscopy and other procedures are important, and a definitive diagnosis is made when characteristic features of either UC or CD are obtained (i.e., when the criteria for diagnosis of either one are satisfied). After observing the progress of IC for 8 years, a definitive diagnosis of CD or UC was made in $80 \%$ of cases [54]. Treatment strategies are decided with a tentative diagnosis based on the clinical and imaging features.

When CD is suspected but without a definitive diagnosis, as in patients with aphthous ulcers that show no longitudinal arrangement and patients who do not have noncaseating epithelioid cell granuloma, regular follow up, using laboratory test results and morphological examinations is necessary to be able to make a definitive diagnosis of $\mathrm{CD}$ when the criteria for the diagnosis are met. Use mainly symptomatic treatments until a definitive diagnosis is made. If morphological examinations cannot confirm the diagnosis, but CD is suspected comprehensively by symptoms and laboratory test results, treatments for CD may be initiated, while observing the patient's progress and conducting follow-up examinations. 


\section{II-9. Determination of severity}

CQ17: How are the severity and activity of the disease determined? [40, 56, 57]

- The severity and activity are usually determined on the basis of clinical symptoms. C1 (Japan VI, overseas VI; 7).

- The IOIBD and CDAI can be used to quantify the disease activity, but these indices are not easy to use in daily clinical practice. C1 (Japan VI, overseas VI; 8).

\section{Comments}

The IOIBD score consists of nine clinical parameters and hemoglobin, and it is a convenient index that is used in the disease datasheet of the Japanese national CD registration. Though the IOIBD data have a certain level of correlation with the CDAI scores [40], the number of items showing correlation is limited, and the IOIBD is not suitable for detailed evaluation of the long-term progress of the disease. In Europe and North America, the CDAI [40], in which eight indices are calculated, is used as a standard index for the assessment of disease activity; it is also used to assess treatment efficacy in clinical studies in Japan. However, calculation of the CDAI requires that clinical symptoms and laboratory test data are available over the 7 days immediately before the day of calculation. Thus, it is not suitable for use in daily clinical practice. Although assessment of severity is important for treatment, severity does not always correlate with disease activity [56]. In some patients, particularly those with smallintestinal lesions, the clinical symptoms are mild, and disease activity is not reflected in the CDAI scores.

In selecting treatment options, comprehensive evaluation should be made. The initial treatment might be modified during the follow-up period. A recent opinion suggests that the disease pattern (whether the case is susceptible to fistulation or stenosis) and the mucosal healing of gastrointestinal lesions should be evaluated.

The European Crohn's and Colitis Organisation (ECCO) has classified disease activity in the categories of mild, moderate, and severe according to the criteria shown in Table 6 [57].

\section{General principles of treatment}

\section{III-1. Outline of treatment}

CQ1: If a patient has a diagnosis of $\mathrm{CD}$, what will the treatments be, and how will this affect the patient's lifestyle? [56-60]

- In the active stage, the treatments are directed to induce remission; once remission is induced, the treatments are given to maintain remission for a prolonged period. $\mathrm{B}^{*}$ (Japan VI, overseas VI; 9).

- Therapeutic modalities include medical treatments, such as drug therapies and nutritional therapies, and surgical treatments. They are selected as monotherapy or combination therapy. B* (Japan VI, overseas: VI 9).

- The majority of patients can live a normal daily life, with regular school life or working hours. In patients with severe or fulminant symptoms, or frequent relapses, the patient needs to be hospitalized, or requires surgical treatment, and faces dietary and lifestyle restrictions. C1 (Japan VI; 8).

\section{Comments}

Repeated remissions and relapses are characteristic during the course of $C D$. Because CD is not curable at present, the goal of the treatment is to control disease activity and to improve the QOL of the patient. For this purpose, it is important to control the symptoms, to maintain nourishment, and to prevent relapse or postoperative recurrence by combining drug therapies, nutritional therapies, and surgical treatments [58].

Treatments are selected according to the sites of lesions, the levels of inflammation, the disease pattern, the responses to treatments in the past, and the presence or absence of complications. Although there is abundant evidence in regard to the efficacy of different treatments in relation to disease severity or location, the patients should be fully instructed about the disorder, and the treatment should be chosen according to the social background and environment of the patients, as well as the individual pathological conditions [56-59].

Table 6 Classification of disease severity

\begin{tabular}{|c|c|c|c|c|}
\hline & CDAI & Complication & $\begin{array}{l}\text { Inflammation } \\
\text { (CRP) }\end{array}$ & Treatment response \\
\hline Mild & $150-220$ & None & Slight rise & \\
\hline Moderate & $220-450$ & $\begin{array}{l}\text { No manifestation of complications such as bowel } \\
\text { obstruction }\end{array}$ & Clear rise & $\begin{array}{l}\text { No response to treatment for mild } \\
\text { CD }\end{array}$ \\
\hline Severe & $>450$ & Bowel obstruction, abscess, and other features & Great rise & Poor treatment response \\
\hline
\end{tabular}

CDAI Crohn's disease activity index, CRP C-reactive protein 
In mild to moderate cases, remission is sufficiently attained with drug and/or nutritional therapies, and the patient can live a normal daily life with maintenance treatment and some attention to daily life. For moderate cases, the patient needs to be hospitalized during periods of relapse, but can otherwise live an almost normal life [60].

\section{III-2. Consultation}

CQ2: Should a CD patient be referred to a specialist for the treatment? [21]

- On many occasions in the management of $\mathrm{CD}$, consultation with a specialist is necessary. B* (Japan VI, overseas VI; 9).

- Consultation is required for nutritional therapy, antitumor necrosis factor (TNF) therapy, failure to maintain remission, and surgical treatment. B* (Japan VI, overseas VI; 9).

\section{Comments}

Except for very typical cases, consultation with a specialist should be considered for any diagnostic difficulty. If the institution is not sufficient for diagnostic investigations, the patient should be referred to a specialist to establish the diagnosis and to determine the extent and severity of the disease [21].

At the initial diagnosis of $\mathrm{CD}$, consultation with a specialist is preferable for education and general guidance. Quiescent cases can be managed by a general practitioner for remission maintenance and follow up.

Steroid-dependency and the administration of immunomodulators or biologic agents are indications for consultation. In cases of intestinal/extra-intestinal complications, the patient should be referred to specialists in the relevant areas.

\section{III-3. Hospitalization}

CQ3: Under what circumstances should a CD patient be hospitalized? [57, 61, 62]

- Consider hospitalization when the patient does not improve on outpatient treatment. B* (Japan VI, overseas VI; 9).

\section{Comments}

If a patient does not improve with outpatient drug or nutritional therapies, and has persistent symptoms such as frequent diarrhea, abdominal pain, fever, and weight loss, and/or elevated inflammatory markers, hospitalization should be considered [57, 61]. In patients with stenosis causing bowel obstruction and those with intra-abdominal abscess formation, hospitalization and surgical treatment should be considered [57]. According to a case series study, a high proportion (50-80\%) of CD patients with small-intestinal lesions require hospitalization/surgery as the disease progresses [62].

\section{III-4. Exercise and social activities}

CQ4: Does a CD patient require rest and restriction of social activities? [31, 60, 63, 64]

- Generally, patients do not require rest or restriction of activities. C1 (Japan VI, overseas VI; 8).

- Patients in the active phase with severe abdominal symptoms, a finding of systemic inflammation, and exhaustion should avoid excessive exercise. C1 (Japan VI; 8).

- In the active phase of the disease, patients face restrictions of social activities, such as school or work, due to treatment or hospitalization. B (Japan IVb; 8).

\section{Comments}

In the long-term progression of $\mathrm{CD}$, the $\mathrm{QOL}$ of the patients is generally well maintained. The number of patients with poor QOL for whom social activities are significantly restricted due to symptoms and/or treatment is limited [31,63].

If remission is maintained, normal exercise, work, and school attendance are expected. However, in the active phase, too much exercise causing a heavy physical or mental burden is to be avoided [31,60]. There is no evidence that bed rest or lifestyle restrictions contribute to the maintenance of remission. Rather, some reports have indicated that moderate exercise reduces $\mathrm{CD}$ disease activity and mental stress [64].

\section{III-5. Diet}

CQ5: Is dietary therapy necessary for the treatment of CD? [21]

- There are no specific dietary therapies to cure or improve CD. C1 (Japan VI, overseas: VI; 9).

- For patients in the active phase, considerinflammation in the gastrointestinal tract when selecting foods, . C1 (Japan VI, overseas VI; 8). 
- Do not allow the patient to take food orally in the presence of severe inflammation or obstruction. C1 (Japan VI, overseas VI; 8).

\section{Comments}

Dietary therapy denotes management in which the amounts of meals or food ingredients are adjusted, with the aim being to overcome or alleviate a disease. Unlike findings with hypertension, hyperlipidemia, and diabetes, no primary therapeutic effect of any dietary therapy in CD has been scientifically proven. Dietary guidance or advice is preferably minimal, such as cautions to avoid excessive drinking and eating or stimulants.

Although the causes of CD are unknown, it is assumed that some dietary factors are involved in the onset and the persistence of inflammation. In many cases, the oral intake of foods exacerbates the symptoms, and these patients have usually had an unbalanced diet before the onset of the disease; thus, it can be presumed that diet may have some relationship to $\mathrm{CD}$. In general, patients with inflammation in the gastrointestinal tract are recommended to avoid fats, stimulants, and dietary fiber. In CD patients, this recommendation also means that the antigens in the diet are reduced to keep the intestinal tract at rest.

Nutritional deficiency is often found in CD patients due to various causes. When $\mathrm{CD}$ is diagnosed, the nutritional condition of the patient should therefore be assessed, and assessment should be carried out regularly during the progress of the disease, with nutritional support being provided according to the patient's pathophysiological condition [21].

CQ6: What kind of general dietary recommendations should be made? [15, 60]

- Basic recommendations during the active phase include having a low-fat, low-residue diet with low levels of stimulants, and food high in protein and calories, to improve the nutritional state while keeping the intestinal tract at rest. C1 (Japan VI, overseas VI; 7).

- In the remission phase, no strict dietary restrictions are necessary, but a low-fat diet is preferred. C1 (Japan IVb; 7).

- The response of the gastrointestinal tract to foods varies considerably from person to person. Accordingly, individual patients should avoid specific foods that make their symptoms worse. C1 (Japan VI, overseas VI; 8).

\section{Comments}

Dietary guidance differs according to the individual and their symptoms. Basically, no foods are absolutely restricted; however, it is advisable that $\mathrm{CD}$ patients maintain an orderly and regular diet, and learn which foods exacerbate their condition, and avoid such foods. Although there is little evidence to show any relationship between the diet and food ingredients and the disease activity of $\mathrm{CD}$, a Japanese case-control study has indicated that fats are a risk factor for CD [15]. Low-fat, low-residue, high-protein, and high-calorie foods are basic dietary suggestions for CD [60]. When a patient undergoes a resection of the small or large intestine, the diet should be considered according to the region that has been resected.

As evidence is lacking for the effects of health food products and popular supplements, and because their safety has not been confirmed, they are not recommended.

\section{III-6. Smoking}

CQ7: Should a CD patient refrain from smoking? [65-71]

- Upon diagnosis, the patient with $\mathrm{CD}$ should quit smoking. B (overseas III; 8).

\section{Comments}

An analytical epidemiological study showed that smoking was associated with the onset of CD [65]. It has also been reported that in infants exposed to passive smoking this has an adverse effect [66]. Case-control studies have indicated that, after remission is induced by medical or surgical treatments, the relapse rates and requirements for surgery are higher among smokers [67-69]. An interventional trial showed that a group that continued to refrain from smoking for more than 1 year had a better prognosis than continuos smoker [70]. With these lines of evidence, smoking cessation is recommended for those who have a diagnosis of CD.

A multivariate analysis of the factors influencing the therapeutic effect of infliximab concluded that smoking was not an independent factor influencing the therapeutic effect; however, for the above-mentioned reasons, patients with $\mathrm{CD}$ are recommended to quit smoking [71].

\section{III-7. Alcohol drinking}

CQ8: Should a CD patient refrain from drinking alcohol? [60]

- It is not necessary to recommend to every patient with $\mathrm{CD}$ that they refrain from drinking alcohol. However, it is preferable to avoid excessive drinking and to stop 
drinking when the disease is in the active phase. $\mathrm{C} 1$ (Japan VI, overseas VI; 7).

\section{Comments}

There is little evidence that drinking alcohol affects the disease activity or progression of CD. However, alcohol may injure the mucosa of the intestinal tract and exacerbate the symptoms of $\mathrm{CD}$. In the remission phase, drinking modest amounts of alcohol is acceptable, but it would be a good practice to advise patients to restrain themselves when drinking, as some people tend to drink excessively.

\section{Therapeutic intervention (Fig. 2)}

\section{IV-1. Treatment options}

CQ1: What are the treatment options for CD, and in what combinations? [56-58]

- Treatment strategies include drug therapies, nutritional therapies, surgical therapies, and other modalities.
Select the most appropriate treatments according to the severity, extent of the lesion, and disease pattern. C1 (Japan VI, overseas VI; 9).

- Initially, or in a relapse, apply drug therapies and nutritional therapies as monotherapy or in combination, with the aim being to induce remission. C1 (Japan VI, overseas VI; 8).

- In patients with intestinal stenosis, fistulas, abscesses, and/or perianal lesions, or in cases that are resistant to medical treatment, consider surgical treatment. C1 (Japan VI, overseas VI; 8).

- Once remission is induced, maintain remission using drug therapies (5-aminosalicylic acid [5-ASA] preparations, immunomodulators, anti-TNF agents), and/or nutritional therapies as monotherapy or in combination. C1 (Japan VI, overseas VI; 9).

\section{Comments}

At present, no treatments can completely cure CD. The purposes of treatment are to control the disease activity and to improve the QOL of the patients; in other words, the aim
Fig. 2 Treatment of Crohn's disease $(C D) .5-A S A$

5-Aminosalicylic acid, $S A S P$ salazosulfapyridine, $T N F-\alpha$ tumor necrosis factor- $\alpha$

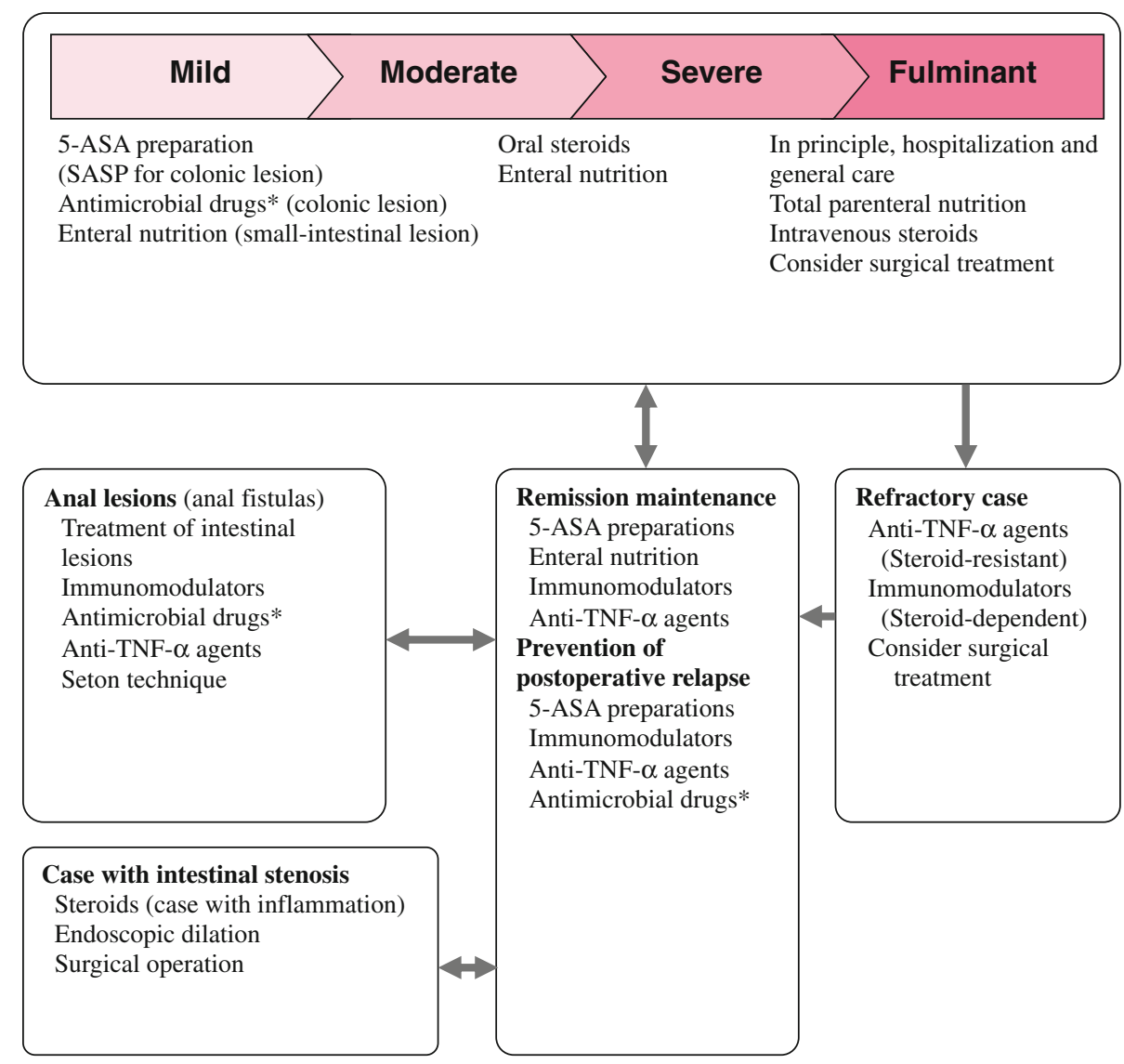

Note: Medical therapies are the standard treatment for CD, but surgical treatment should always be kept in mind while performing treatment.

* Not covered by Japanese public health insurance. 
is to maintain remission for as long as possible. For these purposes, select drug, nutritional, and surgical therapies as indicated to attenuate symptoms, to maintain nourishment, and to prevent relapse. Patients should be fully instructed about the disorder, and the treatment should be chosen according to the social background and environment of the patients, as well as the individual pathological conditions [56-58].

Both drug and nutritional therapies for CD inevitably have adverse effects. However, the benefit of each therapeutic modality outweighs the risk. Some therapies are safe in the short term, but may produce adverse effects in the long term. It is desirable that nutritional therapies and drug therapies be used to supplement each other.

\section{IV-2. Steroids}

CQ2: When are steroids indicated? What kinds of benefits and harms are expected? [21, 59, 72-74]

- Steroids possess potent anti-inflammatory effects. They are effective in inducing remission, but ineffective for maintaining remission. A (Japan V; overseas I; 8).

- Steroids may cause adverse effects, particularly with longterm administration. Thus, steroids should be administered mainly to induce remission, and the dosage should be tapered until they are discontinued. C1 (Japan VI, overseas VI; 8).

- Steroids are indicated for patients with moderate to severe disease activity, as well as for mildly active disease that is refractory to 5-ASA preparations. A (Japan VI, overseas II; 8).

\section{Comments}

Randomized controlled trials were conducted in Europe and North America in the 1970s and 1980s to evaluate the effect of steroids, and a meta-analysis showed their efficacy in inducing remission [72]. However, efficacy in maintaining remission was not shown [73]. In the randomized controlled trials adopted in the meta-analysis, steroids were shown to be more efficacious than placebo or 5-ASA preparations in cases of disease of varying severity, with CDAI scores ranging from 150 to 450 [72]. However, the indication for steroids has changed with the emergence of anti-TNF agents.

While steroids have potent anti-inflammatory effects, they may cause adverse effects such as compromised immune functions, impaired glucose tolerance, delayed wound healing, and osteoporosis. Furthermore, they are not effective in maintaining remission. Accordingly, steroids should not be administered for prolonged periods [21]. In cases where 5-ASA preparations cannot induce remission, oral administration of steroids is recommended. When administering steroids, the dosage should be tapered down to eventual termination irrespective of the response $[21,56,58,59]$. Daily administration of $9 \mathrm{mg}$ budesonide (not yet approved in Japan), which is a steroid with reduced systemic side effects, is effective in inducing remission in mild to moderate cases $[56,57,74]$.

\section{IV-3. 5-ASA preparations}

CQ3: When are 5-ASA preparations indicated? What kinds of benefits and harms are expected? [75-77]

- 5-ASA preparations have clinical efficacy in active CD. A (overseas I; 8).

- During the remission phase, 5-ASA preparations have a limited effect on maintaining remission, but harm is minimal. A (overseas I; 8).

\section{Comments}

According to a meta-analysis of randomized controlled trials, in cases with mild to moderate activity, mesalazine $4 \mathrm{~g} /$ day significantly reduced CDAI scores compared with placebo [75].

Another meta-analysis performed to evaluate the efficacy of 5-ASA preparations in remission maintenance did not show any difference between the 5-ASA preparations and placebo [76]. Another meta-analysis suggested that 5-ASA preparations were significantly efficacious in maintaining remission in $\mathrm{CD}$ [77].

Because the safety profiles of 5-ASA preparations are good, these preparations are frequently used in actual practice. They are also administered in the long term in many patients for the purpose of maintaining remission.

\section{IV-4. Immunomodulators}

CQ4: When are immunomodulators indicated? What kinds of benefits and harms are expected? [78, 79]

- Azathioprine (AZA) and 6-mercaptopurine (6-MP)* are effective in inducing remission in $\mathrm{CD}$, but their adverse effects should be noted. A (overseas I; 8) *Not covered by Japanese public health insurance.

- AZA is effective in maintaining remission in $\mathrm{CD}$, and has a steroid-sparing effect. A (overseas I; 9).

\section{Comments}

Daily administration of $2.0-3.0 \mathrm{mg} / \mathrm{kg}$ AZA, and daily administration of $50 \mathrm{mg}$ (or of $1.5 \mathrm{mg} / \mathrm{kg}$ ) 6-MP, are both useful in inducing remission in $\mathrm{CD}$ in the active phase [78]. 
Daily administration of $1.0-2.5 \mathrm{mg} / \mathrm{kg}$ AZA in patients with quiescent $\mathrm{CD}$ is effective to prevent relapse for 6 months to 2 years. The steroid-sparing effect of immunomodulators is useful for withdrawing steroids. However, it is not clear whether AZA has a long-term effect on remission maintenance [79]. A higher dose $(2.5 \mathrm{mg} / \mathrm{kg}$ daily) of AZA has a more potent effect on remission maintenance than a lower dose $(1.0$ or $2.0 \mathrm{mg} / \mathrm{kg}$ daily). Immunomodulators are slow-acting drugs, and may cause serious side effects (e.g., myelosuppression and pancreatitis.); therefore, the benefits and harms of these drugs should be carefully considered. Because Japanese have a lower ability to metabolize these drugs than Caucasian, Japanese patients are particularly susceptible to the dose-dependent adverse effects of immunomodulators. Smaller doses (AZA 50-100 mg daily) than those used overseas are usually administered in Japan.

\section{IV-5. Anti-TNF agents}

CQ5: When are anti-TNF agents indicated? What kinds of benefits are expected? [80-85]

- Anti-TNF agents are effective to induce remission. A (overseas I; 9).

- In CD patients brought into remission by anti-TNF agents, these agents are also effective for fistula-closure and remission maintenance. A (overseas I; 8).

- When infliximab has not been successful, adalimumab may be effective in inducing remission and attenuating symptoms. A (overseas I; 8).

\section{Comments}

Adalimumab, a humanized anti-TNF- $\alpha$ monoclonal antibody agent, was approved in Japan for the treatment of CD in 2010, in addition to infliximab.

A randomized controlled trial on the remission induction effect of infliximab in patients with active $C D$ indicated that a single administration of $5 \mathrm{mg} / \mathrm{kg}$ was efficacious in inducing remission in CD [80]. Moreover, $5 \mathrm{mg} / \mathrm{kg}$ or $10 \mathrm{mg} / \mathrm{kg}$ of infliximab given every 8 weeks to patients with $\mathrm{CD}$ in remission brought about by infliximab was efficacious in maintaining remission and fistula closure [81, 82]. In clinical practice, $5 \mathrm{mg} / \mathrm{kg}$ of infliximab is administer at week 0 , week 2 , and week 6 , and then at intervals of 8 weeks. If the required effect is not obtained, consider other treatments instead of simply continuing this agent.

A randomized controlled trial on the remission induction effect of adalimumab indicated that two subcutaneous administrations of $80 / 40$ or $160 / 80 \mathrm{mg}$ of this drug in CD patients with moderate disease activity showed a significant remission induction effect at week 4 [83]. A trial on its remission maintenance effect indicated that administration of $40 \mathrm{mg}$ subcutaneously at intervals of 2 weeks or 1 week showed a significant remission maintenance effect at week 56 [84]. In clinical practice, $160 \mathrm{mg}$ of adalimumab is initially administered subcutaneously, followed by $80 \mathrm{mg}$ 2 weeks later, and then $40 \mathrm{mg}$ at intervals of 2 weeks, in order to maintain remission.

In patients with active $C D$ in whom infliximab was unsuccessful (due to intolerance or symptoms persisting after its administration), adalimumab had a significant effect on remission induction and had attenuated symptoms at 4 weeks after administration [85]. That trial, however, did not directly compare adalimumab with infliximab, nor did it deal with remission maintenance.

CQ6: What harms are anticipated with the use of anti-TNF agents? [81, 86-92]

- Cases of serious infections and cases of opportunistic infections have been reported among patients who received infliximab or adalimumab. B (overseas IVa; 8).

- Infliximab increases the chance of tuberculosis infection (including reactivation). B (overseas IVa; 8).

- The occurrence of malignant tumors, including lymphoma, was reported among patients who received infliximab. B (overseas IVa; 8).

- The incidence of cancer in general among patients who received adalimumab does not seem to be different from that in the general population. $\mathrm{C} 1$ (overseas V; 8).

\section{Comments}

A meta-analysis of studies of infliximab indicated no significant difference in the incidence of serious infections between the infliximab and placebo groups [81]. A multivariate analysis of a prospective study of more than 6,000 people in the TREAT Registry suggests that the causes of increases in serious infections in $\mathrm{CD}$ patients are not related to the use of infliximab, but to steroids, narcotic analgesics, and the severity of the disease [86]. In addition, the risk of opportunistic infection in patients with IBD was shown to increase due to the combined use of multiple immunomodulators with infliximab and old age [87].

However, infliximab is known to increase the reactivation and incidence of tuberculosis infection. In addition, it is known that it increases extra-pulmonary lesions and disseminated tuberculosis [88]. Screen patients with CD for tuberculosis infection before administering infliximab, and 
consider the prophylactic administration of anti-tuberculosis drugs, as necessary.

Analysis of the TREAT Registry indicated a worsening of intestinal stenosis in those who received infliximab, but a multivariate analysis concluded that the only risk factors were the duration and severity of the disease, small-intestinal lesions, and the initiation of steroid therapy [89]. Although few studies have indicated a relationship between infliximab and the occurrence of stenosis, patients should be informed of the risk of stenosis after taking infliximab, and surgeons should be notified if a CD patient is receiving this drug.

A further study with the TREAT Registry indicated a significant difference in the incidence of malignant tumors, including lymphoma, between groups with and without the use of infliximab [90]. A multicenter matched-pair study in Italy showed similar results [91]. However, the observation periods in these two studies were not long enough to reach definitive conclusions. Nevertheless, patients should be notified of the risk before infliximab treatment is initiated.

Adverse effects over a period of 10 years were reported in 20,000 patients from 36 clinical studies on six immunemediated diseases [92]. In patients with $\mathrm{CD}$, abscesses in the abdominal cavity or the gastrointestinal tract and opportunistic infections were found, but in general, no risk of major infections was found. The incidence of cancer in these CD patients was equivalent to that in the general population.

The harmful effects noted above are considered to be common to all anti-TNF agents.

\section{IV-6. Antimicrobial drugs}

CQ7: When are antimicrobial drugs indicated, and what kinds of benefits and harms are anticipated? [93-97]

- Antimicrobial drugs* are sometimes effective in attenuating the clinical symptoms of CD. A (overseas I; 8). *Antimicrobial drugs are not covered by the Japanese public health insurance system when used for the treatment of CD.

- These drugs are more effective for colonic lesions than for small-intestinal lesions. A (overseas II; 8).

\section{Comments}

In some cases, antimicrobial drugs such as metronidazole and ciprofloxacin are used for the treatment of CD. Multiple randomized controlled studies have been conducted to evaluate the efficacy of antimicrobial drugs for treating active $\mathrm{CD}$, and the drugs showed efficacy in attenuating the clinical symptoms [93-96]. They were more efficacious for colonic lesions than for small-intestinal lesions [96]. Another report indicated that the administration of antimicrobial drugs was efficacious in preventing the postoperative recurrence of $\mathrm{CD}$ [97]. However, the indications and the specific treatment strategies for antimicrobial therapies for CD have not yet been established.

When antimicrobial drugs are used for a long period of time, caution should be exercised regarding potential adverse effects. Metronidazole, in particular, may cause peripheral neuropathy.

\section{IV-7. Enteral nutriton}

CQ8: When is enteral nutrition indicated, and what kinds of benefits and harms are expected? [98-105]

- The efficacy of enteral nutrition for inducing remission in active $\mathrm{CD}$ is equivalent or slightly inferior to that of corticosteroids. A (Japan III, overseas I; 8).

- Elemental diet therapy is effective in maintaining remission in CD. A (Japan II; 8).

- Although enteral nutrition is safe, maintenance of the patient's acceptance is often difficult. C1 (Japan VI; 8).

\section{Comments}

The results of several randomized controlled studies have indicated that the efficacy of enteral nutrition for remission induction in active $\mathrm{CD}$ is equivalent or slightly inferior to that of corticosteroids [98-102]. A Japanese report indicated that enteral nutrition with an elemental diet had a higher rate of remission induction than prednisolone and was particularly effective for attenuating intestinal lesions [102]. This therapy is safer than corticosteroids.

Enteral nutrition is effective for the maintenance of remission. It was reported that enteral nutrition with an elemental diet for half of the total intake of calories was efficacious in maintaining remission [103]. It was also reported that continuing an elemental diet of $30 \mathrm{kcal}$ per kilogram per day was efficacious in preventing $C D$ relapse $[104,105]$. However, it is frequently difficult to continue enteral nutrition for an extended period because its acceptability to the patient decreases.

CQ9: Are there differences in the therapeutic effect between oligomeric and polymeric nutrients?

$[98,99,106,107]$

- There are no significant differences between oligomeric and polymeric nutrients in terms of their efficacy in remission induction for active CD. A (Japan III, overseas I; 7). 


\section{Comments}

Oligomeric nutrients are enteral formulas which have amino acids and oligopeptides as a nitrogen source, with a lowcontent of fats, and these formulas are therefore easily digested and assimilated. Among oligomeric nutrients, an elemental diet has amino acids as a nitrogen source, and contains little fat. Polymeric nutrients have proteins as a nitrogen source and contain some fats. Polymeric nutrients contain various nutrients in a good balance, and are easily taken orally. Many randomized controlled studies have been conducted to examine the differences among various enteral nutrients in terms of their therapeutic efficacy for active CD. The results have indicated no significant differences in the effect of remission induction between oligomeric and polymeric nutrients [98, 99, 106, 107]. In Japan, there are some opinions that oligomeric nutrients are clinically superior to polymeric nutrients.

CQ10: When is it necessary to administer enteral nutrients through a nasogastric tube?

- A nasogastric tube is necessary in cases where enteral nutrients should be given at a fixed rate, the oral intake of such nutrients is difficult, or such nutrients need to be given to patients at home during sleep at night. $\mathrm{C} 1$ (Japan VI, overseas VI; 8).

\section{Comments}

Enteral nutrients for patients with CD are taken orally, or through a nasogastric tube inserted into the stomach or duodenum. Unpalatable oligomeric nutrients are difficult to take orally, and in many cases are given through a nasogastric tube. Enteral nutrient feeding through a nasogastric tube at a fixed rate, using an enteral feeding pump, causes fewer side effects (such as diarrhea or abdominal pain) than orally taken enteral nutrients. For home enteral feeding, it is possible to give enteral nutrients to a patient through a nasogastric tube at night, while the patient is sleeping.

\section{IV-8. Parenteral nutrition}

CQ11: When is parenteral nutrition indicated, and what kinds of benefits and harms are expected? [58, 107-110]

- Total parenteral nutrition (TPN) is indicated for patients with active $\mathrm{CD}$ who have serious malnutrition, frequent diarrhea, and/or a critical disease state with extensive small-intestinal lesions, or in patients who have severe stenosis in the intestinal tract, fistulas, abscess formation, massive hemorrhage, and/or severe perianal lesions. The patient must be fasting when parenteral nutrition is administered. C1 (Japan VI, overseas VI; 8).

- Total parenteral nutrition (TPN) is efficacious in inducing remission in active $\mathrm{CD}$, and has a therapeutic effect equivalent to that of enteral nutrition. B (Japan III, overseas III; 8).

- When carrying out TPN, watch for complications such as sepsis and hepatic disorders. C1 (overseas V; 8).

\section{Comments}

The indications for TPN through a central venous line are described in the Proposed revision of the clinical practice guidelines for Crohn's Disease published by Research Group of Intractable Inflammatory Bowel Disease subsidized by the Ministry of Health, Labour and Welfare of Japan [58]. However, in practice, it is necessary to select a feeding method according to the individual's condition. The results of several randomized controlled studies have shown that TPN, among other parenteral feeding methods, has a remission induction effect for active $\mathrm{CD}$ equivalent to that of the enteral feeding of elemental nutrients [107, 108]. TPN can also attenuate intestinal lesions [108, 109]. When the CD patient's condition becomes stable on TPN, the TPN can be switched to enteral feeding.

If TPN is provided through a central venous line, catheter-related complications such as sepsis and hepatic disorders could occur. Particularly in patients undergoing parenteral nutrition at home through a central venous line, an infected feeding port is likely to progress to sepsis [110].

\section{IV-9. Cytapheresis}

CQ12: When is cytapheresis indicated, and what kinds of benefits and harms are expected? [111]

- Cytapheresis is indicated in patients with active CD with colonic involvement in whom drug and/or nutritional therapies are ineffective or inapplicable; the addition of granulocyte-monocyte apheresis (GMA) may accelerate induction of remission. C1 (Japan V; 7).

\section{Comments}

Cytapheresis has become an established therapeutic modality for UC in Japan. For CD, the effects of GMA in 
combination with other therapies were studied in 21 cases refractory to existing drug and/or nutritional therapies. The results showed remission (i.e., CDAI less than 150) in $27.8 \%$, and improvement (i.e., reduction of CDAI by 50 or more) in $16.7 \%$ [111]. GMA was approved for clinical use in CD with colonic lesions in Japan in 2010. Although some adverse events, such as headache, dizziness, palpitation, and minor nonspecific abnormal laboratory test results have been reported, these therapies are generally considered to be safe.

\section{IV-10. Surgical treatment}

CQ13: What kind of benefits and harms are expected with surgical treatment? [112-114]

- Surgical treatment for the complications of $C D$ is expected to attenuate the symptoms and improve the QOL. B (overseas IVa; 9).

- Surgical treatment reduces the doses of therapeutic drugs and thus the possibility of adverse effects. C1 (Japan V, overseas IVa; 7).

- Surgical treatment involves the risk of postoperative complications such as short-bowel syndrome and anastomotic leaks. C1 (Japan V, overseas V; 8).

\section{Comments}

There are no therapies that can cure CD completely. The purpose of surgical treatment is to attenuate the symptoms due to complications that are the causes of the patient's disability, and to improve the patient's QOL [112]. If the symptoms are attenuated, the doses of therapeutic drugs such as steroids can be reduced, thus preventing possible adverse effects (such as growth retardation) [113]. However, reoperation becomes necessary in some cases (the rate of reoperation in Japan was reported to be as high as 28-30 \%) [114]. Short-bowel syndrome due to repeated bowel resections can compromise the patient's QOL; resection should therefore be kept to the minimum necessary.

\section{IV-11. Endoscopic treatment}

CQ14: When is endoscopic balloon dilation indicated, and what kinds of benefits and harms are expected?

$[115,116]$

- Endoscopic balloon dilation is indicated for benign stenosis with bowel obstruction in the gastrointestinal tract without accompanying deep ulcers or fistulas. $\mathrm{C} 1$ (Japan VI, overseas VI; 7).

- This therapy may alleviate bowel obstruction and may avoid a surgical operation. C1 (Japan V, overseas V; 8).

- Care must be taken with regard to complications such as perforation or restenosis. C1 (Japan V, overseas V; 9).

\section{Comments}

The major endoscopic treatment for $\mathrm{CD}$ is endoscopic balloon dilation (EBD). In CD, EBD is indicated for benign stenosis of comparatively short length with few flexures producing clinical features of bowel obstruction, without deep ulcers or fistulas. Accordingly, it is necessary to employ endoscopy or contrast imaging to observe the stenosis well before EBD is performed. The effectiveness of EBD for CD has been reported from many medical institutions in Japan and overseas $[115,116]$. It is particularly effective for stenosis of a comparatively short length, such as $4 \mathrm{~cm}$ or less [115]. The incidence of complications involved in EBD was $2 \%$, and perforation accounted for most of the cases [116]. Other than perforation, care must be taken with regard to hemorrhage, fistulas, abscess formation, and further restenosis.

\section{Active phase treatment}

\section{V-1. Mild to moderate}

CQ1: How is treatment initiated for mildly to moderately active $\mathrm{CD}$ ? $[56,75,98,99,102$, 117-124]

- Administer salazosulfapyridine (SASP) in patients with mildly to moderately active $\mathrm{CD}$ with colonic lesions. A (overseas II; 8).

- SASP is not effective for small-intestinal lesions. B (overseas III; 8).

- The effect of 5-ASA preparations is limited, but they lack serious side effects and are easy to administer. In practice, they are often chosen as a first-line drug. A (overseas I; 8).

- Daily administration of $1,000 \mathrm{mg}$ ciprofloxacin* is expected to have an effect similar to that of 5-ASA preparations for colonic lesions in CD. B (overseas III; 7) *Not covered by Japanese public health insurance for treatment of CD.

- The remission induction effect of enteral nutrition for active $\mathrm{CD}$ is equivalent or slightly inferior to that of corticosteroids. A (Japan III, overseas I; 8). 


\section{Comments}

As described in section II-9, there are no practical criteria to determine the severity of $\mathrm{CD}$, and thus it is determined on the basis of a comprehensive assessment of clinical findings [56]. In these guidelines, mild to moderate cases are assumed to be those in which the patient is capable of visiting a hospital on an outpatient basis and of taking food orally, without findings including dehydration, fever, abdominal tenderness, bowel obstruction, or weight loss of $10 \%$ or more [56].

A meta-analysis and several randomized controlled trials have shown that SASP is effective for colonic lesions in mildly to moderately active $\mathrm{CD}[117,118]$, but that it is not effective for small-intestinal lesions [119]. Some studies have also shown that mesalazine is effective for CD [75, 120-122]. A recent meta-analysis indicated that the CDAI score in the mesalazine group was significantly reduced in comparison with that in the placebo group [119]. In Japan, where treatment options are limited, mesalazine is widely used for ileal and colonic lesions because of its safety profile and ease of administration.

A randomized controlled trial has indicated that for mildly to moderately active CD, 1 g ciprofloxacin administered daily has an effect similar to that of $4 \mathrm{~g}$ mesalazine administered daily [123].

Meta-analyses that compared enteral nutrition with steroid therapy concluded that steroids were more efficacious [98, 99]. However, a randomized controlled small-size study has indicated that nutritional therapy using elemental nutrients has an effect of remission induction similar to that of steroids (prednisolone $0.5 \mathrm{mg} / \mathrm{kg}$ daily), and provides better nutritional status than steroids [124]. Furthermore, a Japanese report indicated that enteral feeding of elemental nutrients exhibited a higher rate of remission induction than prednisolone treatment, particularly for intestinal lesions [102]. In Europe and North America, budesonide, a steroid with reduced systemic adverse effects, is used for lesions in the ileum to the right side of the colon. Budesonide exhibited a higher rate of remission induction than mesalazine.

\section{V-2. Moderate to severe}

CQ2: How is treatment initiated for moderately to severely active CD? [56, 73, 111, 119, 125-128]

- Administer oral steroids (prednisolone, approximately $40 \mathrm{mg}$ daily). B (overseas III; 8).

- In cases where steroids are not effective, consider administering an anti-TNF agent. A (overseas II; 8).

- The remission induction effect of enteral nutrition for active $\mathrm{CD}$ is equivalent or somewhat inferior to that of corticosteroids. A (Japan III, overseas I; 8).
- In active CD with colonic lesions for which drug and/or nutrition therapies are ineffective or inapplicable, the addition of GMA may be helpful. C1 (Japan V; 7).

\section{Comments}

In these guidelines, moderate to severe cases are assumed to be those in which therapies for mild to moderate cases are not effective, or those in which the patient exhibits symptoms that include weight loss of $10 \%$ or more, anemia, abdominal pain, and/or nausea/vomiting without bowel obstruction [56].

Randomized controlled trials have shown that steroids are efficacious in inducing remission in CD [73, 119]. However, steroids are not effective in maintaining remission [73]. In patients in whom symptoms have worsened during the tapering of steroids, or those in whom there was a relapse shortly after withdrawal from steroids, or in those with repeated relapses, consider using an immunomodulator such as AZA or 6-MP in combination with steroids. In patients in whom AZA or 6-MP cannot be used because of adverse effects, methotrexate (MTX) is considered to be effective and is used overseas [125, 126].

Anti-TNF agents, both with single administration and with scheduled successive administrations, were shown to be efficacious in cases refractory to steroids and/or immunomodulators [127, 128].

The addition of GMA was approved for clinical use in Japan in 2010 for active CD with colonic lesions not responsive to existing drug and/or nutrition therapies [111].

\section{V-3. Severe to fulminant}

CQ3: How is treatment initiated for severe to fulminant CD? [56, 129]

- The patient is generally hospitalized; as necessary; consider complete fasting, infusion of fluid, and/or blood transfusion, and administer an antimicrobial drug if the patient shows signs of infection. C1 (Japan VI, overseas VI; 8).

- Exclude infections, and intravenously administer steroids (prednisolone equivalent to $40-60 \mathrm{mg}$ daily). C1 (Japan VI, overseas VI; 8).

- In cases resistant to steroids, consider administering an anti-TNF agent. C1 (overseas V; 8).

- In cases where the patient's general condition is poor, or unresponsive to medical therapies, consult with surgeons at an early opportunity. C1 (Japan VI, overseas VI; 8). 


\section{Comments}

In these guidelines, severe to fulminant cases are assumed to be those in which the patient's symptoms persist after oral administration of steroids, or those in which the patient presents with high fever, persistent vomiting, bowel obstruction, rebound tenderness, cachexia, and/or abscess [56].

In general, the patient should be hospitalized to receive intensive general supportive care. Orally administered steroids are not as well assimilated as intravenous steroids, and intravenous administration is advantageous in terms of pharmacokinetics [129] and preferred for severe cases.

For fulminant cases not responsive to other medical treatments, there is only limited evidence on the effects of anti-TNF agents; however, they can still be a treatment option. They should be used only when the presence and/or risk of infectious complications such as abscesses are ruled out. Severe cases in which the patient has unstable hemodynamics, or those with peritoneal irritation, may be indications for surgical treatment, and therefore it is desirable to consult with surgeons at an early opportunity.

\section{V-4. Therapies according to disease extent}

CQ4: Are different therapies used for lesions in the small intestine, the colon, and both? [117, 118]

- Treatment options vary according to the disease extent. C1 (Japan VI, overseas VI; 8).

- SASP is effective only for colonic lesions. A (overseas II; 8).

- Antimicrobial drugs* are more effective for colonic lesions than for small-intestinal lesions. B (overseas III; 8). *Not covered by Japanese public health insurance for treatment of CD.

- Enteral nutrition is more effective for small-intestinal lesions than for colonic lesions. A (Japan III, overseas $\mathrm{I} ; 8)$.

\section{Comments}

Consideration of the mechanisms and sites of action of therapeutic drugs for $\mathrm{CD}$ indicates the appropriate use of these drugs depending on the sites of the lesions, and it is actually confirmed in some cases. SASP is effective only for mild colonic lesions [117, 118]. Antimicrobial drugs, particularly metronidazole, are known to be generally effective for colonic and perianal lesions. Enteral nutrients are significantly more effective for small-intestinal lesions than for colonic lesions. Mesalazine is effective for both small-intestinal and colonic lesions; and systemic steroids, immunomodulators, and anti-TNF agents are not selective with respect to lesion sites.

CQ5: What is the treatment for CD lesions in the upper gastrointestinal tract? [56, 57]

- In cases of CD with upper-gastrointestinal lesions, administer a proton pump inhibitor (PPI). * $\mathrm{C} 1$ (overseas VI; 7) *Not covered by Japanese public health insurance for treatment of CD.

- Administer steroids and/or immunomodulators, such as AZA or 6-MP, as necessary. C1 (overseas VI; 7).

- In cases refractory to steroids, consider administering anti-TNF agents. C1 (overseas: VI; 8).

- In patients with upper-gastrointestinal lesions with bowel obstruction, consider endoscopic dilation or surgical treatment. C1 (overseas VI; 8).

\section{Comments}

Evidence is not sufficient with regard to the treatment for upper-gastrointestinal lesions in active CD. PPIs are often used for inflammatory lesions in the upper gastrointestinal tract, in combination with other therapies, as in the treatment for lesions at other sites [56, 57]. 5-ASA preparations in their original forms do not act on the mucosa of the upper gastrointestinal tract, and therefore attempts have been made to administer these agents orally by crushing and grinding the tablets. However, the effectiveness and safety of these preparations have not been sufficiently studied.

\section{V-5. Perianal lesions}

CQ6: What is the treatment for the perianal lesions of CD? [36, 82, 130-132]

- Treat the intestinal lesions first, and wait to see if the perianal lesions are attenuated. C1 (Japan VI, overseas VI; 8).

- Anti-TNF agents are effective as medical therapy for anal fistulas. A (overseas II; 8).

- Antimicrobial drugs and immunomodulators are effective therapies for anal fistulas. A (overseas I; 8).

- The Seton procedure is an effective surgical treatment for anal fistulas. In severe cases, consider a stoma. B (Japan V, overseas V; 9).

\section{Comments}

Perianal lesions in CD include primary lesions (fissures and cavitating ulcers), ulcerated piles with longitudinal ulcers, 
and secondary refractory lesions (perianal abscesses, anal fistulas). In patients with perianal lesions specific to $C D$, first employ medical therapies and/or surgical treatment for the intestinal lesions, and wait to see if the perianal lesions are attenuated. Among the secondary perianal lesions in $\mathrm{CD}$, the Seton procedure is effective for anal fistulas; for common lesions, use ordinary treatments [130, 131]. An overseas randomized controlled trial indicated that an antiTNF agent was effective for anal fistulas [82]. Anti-TNF agents should be used after confirmation that any infection is under control. There are no randomized controlled studies on antimicrobial drugs (such as metronidazole) for perianal lesions; however, limited evidence and clinical experience indicate that these drugs have some efficacy. For immunomodulators (such as AZA), several randomized controlled trials and meta-analyses have shown effectiveness for anal fistulas [36, 132].

\section{V-6. Refractory cases}

CQ7: What is the treatment for cases refractory to various medical treatment? [57, 133]

- Consider surgical treatment in cases refractory to medical treatment without attenuation of complications. B* (Japan VI, overseas VI; 9).

- The indication for surgical treatment should be determined with mutual communications among the gastroenterologist, the surgeon, and the patient. B* (Japan VI; 9).

\section{Comments}

Medical treatment is the primary therapy for $\mathrm{CD}$, and surgical treatment remains as secondary. However, in cases refractory to medical treatment, in which the patient has extremely impaired QOL, with serious adverse reaction to drugs, or with a fibrous stenosis not expected to be improved by medical treatment, surgical treatment should be considered $[57,133]$. The indication for the surgical treatment should not be determined only by the surgeon, but by thorough discussions involving the relevant healthcare providers together with the patient.

\section{V-7. Fistulas}

CQ8: What is the treatment for fistulas? [82, 132, 134]

- Immunomodulators are effective for treating fistulas, but their onset of the action is delayed. A (overseas I; 8).

- Anti-TNF agents are effective for treating fistulas. A (overseas II; 9).
- Surgical treatment is indicated in patients with internal fistulas causing severe malabsorption. B (Japan VI, overseas VI; 8).

- Consider surgical treatment for fistulas with abscess formation. B*(Japan VI, overseas IV:9).

\section{Comments}

Some cases of CD are complicated with internal fistulas such as intestinal fistulas and external fistulas such as intestinal-cutaneous fistulas. No consensus has been reached with regard to either treatments for fistulas without symptoms or treatments for internal fistulas [134].

As a medical treatment for fistulas, a meta-analysis of overseas randomized controlled studies has indicated that immunomodulators are effective [132], although the onset of action of these drugs is delayed. An overseas large randomized controlled study (ACCENT II) demonstrated that infliximab was useful [82]. A study of the effect of adalimumab on remission maintenance showed a significant effect in closing external fistulas completely at week 26 and week 56.

Consider surgical treatment in patients in whom medical therapies have been unsuccessful. The indications for surgery include patients with internal fistulas causing severe malabsorption, patients in whom fistulas are spread over a broad area in the healthy bowel, patients with repeated urinary tract infections, patients with external fistulas with excessive leakage of intestinal juices, and patients with painful perianal external fistulas complicated with abscess formation [134].

\section{V-8. Stenosis}

CQ9: What is the treatment for an intestinal stenosis due to $\mathrm{CD}$ ? [115, 135, 136]

- Administer steroids in patients with severe inflammation. C1 (Japan VI, overseas VI; 7).

- Consider endoscopic dilation in patients in whom there has been no improvement with drug therapies or decompression. C1 (Japan V, overseas V; 7).

- Consider surgery in patients whose condition has not been improved with medical treatment. B* (Japan V, overseas $\mathrm{V}$; 9).

\section{Comments}

Intestinal stenosis occurs with mucosal edema due to acute inflammation or transmural fibrotic changes in the 
intestine. Patients with stenosis mainly due to inflammation may improve with medical treatment such as steroids [135]. In patients that do not show improvement with anti-inflammatory treatments, suspect stenosis due to fibrosis, and consider the possibility of endoscopic dilation on the basis of the length of the stenosis, the number of stenotic sites, and the presence of ulcers. It is advisable to use endoscopic dilation when the inflammation and ulcers have disappeared or become reduced with enteral nutrition or other therapies. In a report overseas, endoscopic dilation brought about favorable results in $40 \%$ of the subjects [136]. A report from Japan showed the 5-year operation-free rate after endoscopic dilation was $58 \%$ [115]. Whether or not anti-TNF agents are indicated for cases of $\mathrm{CD}$ with stenosis has not yet been determined.

\section{V-9. Hemorrhage}

CQ10: What is the treatment for hemorrhage from the CD lesions? [137-140]

- First apply conservative management such as supportive care and drug therapies. C1 (Japan V, overseas V; 9).

- Infliximab was reported to be effective in arresting hemorrhage. C1 (Japan V, overseas V; 8).

- If conservative management is not successful in arresting hemorrhage, surgery is indicated. B* (Japan $\mathrm{V}$, overseas $\mathrm{V} ; 9$ ).

\section{Comments}

Massive hemorrhage may occur in CD, although it is rare. In such cases, first perform intensive conservative management, and allow nothing by mouth to keep the intestinal tract at rest. It was reported that steroids were efficacious in such cases. Try endoscopic hemostasis where it is applicable. With regard to angiography, it was reported that the intraarterial injection of vasopressin and arterial embolization were successful in arresting hemorrhage [137]. However, arterial embolization may cause intestinal ischemia leading to intestinal necrosis. It was reported that the administration of infliximab was effective for treating the hemorrhagic type of CD [138].

Surgical treatment is necessary in cases where medical treatment is unsuccessful. It was reported that the surgical operation rate for hemostasis in patients with initial massive hemorrhaging was $20-90 \%$, and that the surgical operation rate in those with recurrent hemorrhage under medical treatment was 30-35\% [139, 140].

\section{V-10. Abscesses}

CQ11: What diagnosis and treatment are used for abscesses due to CD? [141-144]

- Imaging examinations such as CT, US, and MRI are used to diagnose abscesses. B (JapanV, overseas IVb; 9).

- Where possible, perform image-guided (e.g., CTguided) percutaneous drainage. B (Japan V, overseas IV; 8).

- In patients with abscesses in the perianal region, perform incision and drainage. B* (Japan V, overseas $\mathrm{V} ;$ 9).

- In patients in whom abscesses recur after percutaneous drainage or in those with fistulas, surgical treatment is likely to be necessary. B* (Japan V, overseas V; 8).

\section{Comments}

In $\mathrm{CD}$, abscesses may be found to be complicated with transmural lesions in the intestinal wall. A Japanese report has indicated that the frequency of such abscesses is approximately $10 \%$ [141]. CT, MRI, and ultrasonographic examination are useful in diagnosing abscesses [142]. For treatment, percutaneous drainage should be performed where possible. The drainage techniques include CT-guided percutaneous drainage, US-guided percutaneous drainage, or surgical drainage via a small incision. In patients having percutaneous drainage, administer an antimicrobial drug with a broad spectrum. Some overseas reports have indicated that percutaneous drainage avoided subsequent surgical operation in $50-69 \%$ of the patients treated [143, 144]. Patients with abscesses that cannot be controlled by percutaneous drainage require surgical treatment.

\section{V-11. Extra-intestinal complications}

CQ12: What is the treatment for extra-intestinal complications of CD? [145-147]

- In patients with active intestinal lesions, treat the intestinal inflammation. C1 (Japan V, overseas V; 8).

- In patientss with pyoderma gangrenosum or uveitis, administer steroids. C1 (Japan V, overseas V; 8).

- In patients with extra-intestinal complications, the usefulness of infliximab was reported. A (overseas II; 8).

\section{Comments}

Extra-intestinal complications in $\mathrm{CD}$ include those related to the activity of the intestinal lesions (e.g., some types of 
peripheral arthritis, erythema nodosum, episcleritis, and intraoral aphthous ulceration) and those unrelated to the activity of the intestinal lesions (pyoderma gangrenosum, uveitis, sacral arthritis, and ankylosing spondylitis). For either category, it is necessary to intensively control the inflammation of the intestinal lesions.

In patients with arthritis, a 5-ASA preparation such as SASP is the first choice. Avoid NSAIDs because they may exacerbate the intestinal lesions. Administer steroids for serious complications such as pyoderma gangrenosum and uveitis.

Some randomized and non-randomized controlled studies, including those with non-CD patients, have indicated that infliximab was effective for treating complications such as pyoderma gangrenosum, arthritis, uveitis, and ankylosing spondylitis [145-147].

\section{Remission maintenance treatment}

\section{VI-1. General principles for preventing relapse}

CQ1: Are there any lifestyle factors that require attention to prevent relapse? [18, 70, 148, 149]

- If the patient smokes, advise them to refrain from smoking. B (overseas III; 9).

- Advise the patient to avoid irregular lifestyle and eating habits, and to refrain from excessive alcohol drinking. C1 (overseas VI; 7).

- Advise the patient to adopt a lifestyle without excessive mental stress, to have as little stress as possible. C1 (overseas IVb; 7).

- Advise the patient that in using an analgesic or an antipyretic, wherever possible to avoid NSAIDs. C1 (overseas IVb; 7).

\section{Comments}

Although exacerbating factors common to all cases of CD cannot be specified, it has been shown that smoking contributes to the disease becoming refractory or relapsing [148], and that the disease is attenuated after smoking cessation [70]. Frequent or excessive alcohol drinking may damage the intestinal tract, and therefore drinking should be controlled. In view of the fact that nutritional therapies are beneficial for $\mathrm{CD}$, irregular eating habits or unbalanced diets may precipitate relapse.

It has also been shown that mental stress has some relationship with CD relapse [149]. Patients with CD should be advised to live without mental stress as much as possible, and to adopt a lifestyle where stress does not accumulate. NSAIDs are known to cause gastrointestinal injuries, and also the relapse or exacerbation of $\mathrm{CD}$. Therefore, wherever possible, they should be avoided. If analgesics or antipyretics need to be prescribed, acetaminophen would be an appropriate substitute [18].

CQ2: Are there any distinctive features of $\mathrm{CD}$ that make the disease likely to relapse? [31, 150]

- CD patients with fistulas, perforation, or perianal lesions, and those who have had resection of the intestinal tract, are more susceptible to relapses. $\mathrm{C} 1$ (Japan V, overseas V; 7).

- Patients with $\mathrm{CD}$ who have required steroids for induction of remission are more susceptible to relapses. C1 (overseas IV; 7).

\section{Comments}

The pathophysiology and symptomatology of $\mathrm{CD}$ are complex, and it is difficult to predict the variable progress of each case. However, it has been shown that patients with fistula formation or intestinal perforation are more susceptible to relapses than those with the non-perforation type [150]. Patients with CD with high disease activity who have required steroids for the induction of remission often have difficulty in withdrawing from the steroids and require immunomodulators. It has been shown that patients for whom steroids or immunomodulators are required are more susceptible to relapses than other patients [31].

\section{VI-2. Drug treatment}

CQ3: Which drugs are effective for maintaining remission of CD? [76, 79, 128, 151-153]

- AZA is effective in maintaining remission. A (overseas I; 9).

- In patients in whom remission was induced by antiTNF agents, the scheduled administration of anti-TNF agents is effective in maintaining remission. A (overseas II; 8).

- 5-ASA preparations are effective in maintaining remission postoperatively. B (overseas II; 7).

\section{Comments}

Steroids are effective for inducing remission, but ineffective for maintaining remission. It has been shown that AZA 
and 6-MP have steroid-sparing effects in steroid tapering and complete withdrawal, as well as having a long-term effect in maintaining remission. The standard dose of AZA is $1.0-2.5 \mathrm{mg} / \mathrm{kg}$ daily, and that of $6-\mathrm{MP}$ is half of that of AZA. A high dose of these drugs exhibits a more potent effect than a low dose [79, 151]. However, both AZA and 6-MP may produce serious side effects, and the doses that may produce the required effects and the adverse reactions differ individually. The recommended doses for these agents have been determined for patients in Western countries, and it is possible that lower doses in Japanese patients would produce the required effects and/or fewer adverse effects.

It has been shown that infliximab exhibits a remission induction effect even in refractory or severe cases, and that administration of infliximab every 8 weeks to patients in whom remission was induced by the agent had a significant effect in preventing relapses for at least 1 year $[128,152]$. 5-ASA preparations have only a limited remission maintenance effect [76], but they are effective in controlling postoperative relapses [153].

CQ4: How long should the treatment for remission be continued? [128, 152, 154]

- If effective, it is advisable to continue the administration of AZA or 6-MP* for 3-4 years. C1 (overseas VI; 8) *Not covered by Japanese public health insurance for treatment of CD.

- Scheduled administration of infliximab is effective for at least 1 year. A (overseas II; 8).

\section{Comments}

AZA and 6-MP, thiopurine derivatives, are known to have efficacy for long-term remission, and a meta-analysis showed that they were effective in maintaining remission for at least 1 year. Furthermore, it has been reported that the continued administration of these agents for more than 2 years is effective, and therefore it is advisable that they continue to be administered for 3-4 years as long as remission is maintained without the emergence of adverse effects [154]. In a study to evaluate the effect of the scheduled administration of infliximab at 8-week intervals for 1 year in patients in whom remission was induced by infliximab, the remission maintenance effect was significantly higher in the group receiving infliximab regularly at intervals of 8 weeks compared with placebo $[128,152]$.

\section{VI-3. Nutritional therapy}

CQ5: Is home enteral nutrition effective in maintaining remission? [103, 155, 156]

- Replacing half of the daily caloric intake by enteral nutrients is effective in maintaining remission. A (Japan II, overseas II; 8).

\section{Comments}

Enteral nutrition as a long-term therapy for remission maintenance has an excellent safety profile, but often lacks acceptability and convenience. It is thus difficult for patients to receive total enteral feeding at home for a long period of time. Partial enteral feeding is more acceptable and more convenient for the patient, and the patient can enjoy eating as well. It was shown that replacing 30-50\% of the normal daily caloric intake by enteral nutrients had a significantly higher remission maintenance rate than a normal daily diet only $[103,155,156]$.

CQ6: How long should nutritional therapy be continued? [103, 155, 156]

- Replacing half of the daily caloric intake by enteral nutrients is effective in maintaining remission for at least one year. A (Japan II, overseas II; 8).

\section{Comments}

It was shown that replacing 30-50\% of the daily caloric intake by enteral nutrients had a significantly higher rate of remission maintenance at 1 year than a normal daily diet only $[103,155,156]$; therefore, it is recommended to continue enteral nutrition for 1 year in patients in whom remission was induced by nutritional therapy. Although there is no evidence to favor enteral nutrition beyond 1 year, it may be advisable to continue this treatment for as long as possible, if no problems of acceptability or convenience emerge.

CQ7: When is home parenteral nutrition (HPN) necessary, and how is it performed? [157]

- In patients with short-bowel syndrome for whom sufficient nutritional care by enteral feeding is not possible, supply nutrition by infusion through a central venous line. C1 (Japan VI, overseas VI; 8). 


\section{Comments}

In $\mathrm{CD}$ patients with small-intestinal lesions where the remaining small intestine is short as a result of the resection of a large area or frequent resections, the bowel cannot digest and assimilate a sufficient amount of nutrition (i.e., if the remaining small intestine is $1 \mathrm{~m}$ or less, malabsorption is inevitable; even if the remaining small intestine is a little longer, malabsorption is likely to occur). Accordingly, to provide the patient with the required nutritional support, a catheter is placed in the central vein, and a home-based arrangement is made whereby the patient or the patient's family can manage nutrition drips [157].

\section{Surgical treatment}

\section{VII-1. Indication for surgery}

CQ1: How often is surgical treatment required for CD? $[158,159]$

- In Japan, the cumulative rate of surgical intervention for $\mathrm{CD}$ at 5 and 10 years after the onset is 30.3 and $70.8 \%$, respectively. The rates vary greatly from area to area in Europe and North America. C1 (Japan V, overseas $\mathrm{V} ; 7$ ).

\section{Comments}

In Japan, the cumulative rates of surgical treatment for CD at 5 and 10 years after the onset are 30.3 and $70.8 \%$, respectively $(N=361)$. Regarding the rate according to the disease location, there were no significant differences among the ileal, ileocolonic, and colonic types at 5 and 10 years after the disease onset [158]. In Europe and North America, the rate of surgical treatment for $\mathrm{CD}$ varies from area to area [159].

CQ2: What are the absolute indications and the relative indications for surgery? [141, 160, 161]

- Surgery is absolutely indicated in patients with perforation, massive hemorrhage, development of cancer, bowel obstruction not alleviated by medical therapies, and abscesses. B* (Japan V, overseas V; 9).

- Surgery is relatively indicated in patients with refractory stenosis or internal and external fistulas, and in those refractory to medical treatment, or with refractory extra-intestinal complications (e.g., growth retardation, pyoderma gangrenosum), and refractory perianal lesions. C1 (Japan V, overseas: V; 8).

\section{Comments}

Surgical indications in CD, in percentages according to the underlying lesions or clinical situations, are as follows: bowel obstruction and stenosis: $54 \%$; fistulas: $28 \%$; abscesses: $7 \%$; perforations: $4.5 \%$; cases refractory to medical therapies: $3.5 \%$; massive hemorrhage: $2 \%$; and colorectal cancer: $1 \%$. Toxic megacolon was also referred to as a surgical indication. Other surgical indications are: symptomatic fibrotic stenosis; enterocutaneous fistulas with excessive leaks of intestinal juices or with stenosis; symptoms due to bypass formation (e.g., duodenal/transverse colonic fistulas); intestinal fistulas involving a broad area of intact bowel; enterovesical fistulas refractory to medical therapies and with repeated urinary tract infection; intra-abdominal abscesses not responsive to medical therapies; and retroperitoneal abscesses [141]. The relative risks of colorectal cancer and small-intestinal cancer are significantly high in patients with CD [161]. In Japan, colorectal cancer in $\mathrm{CD}$ patients is more common in the form of rectal and anal fistula cancer, and small-intestinal cancer is more common in the ileum. With regard to gastric/duodenal lesions as complications of $\mathrm{CD}$, surgical indications are fistulas starting at the colon or ileo-colonic anastomosis to the stomach, long duodenal stenosis, and duodenal fistulas that often occur arising from the adjacent lesions [160].

\section{VII-2. Refractory to medical treatment}

CQ3: What is the main principle guiding surgery on the intestine? [162, 163]

- As CD involves the entire intestinal tract and often recurs, the intestinal tract should be preserved as much as possible. A (overseas II; 9).

- Only the small portion of the intestinal tract causing stenosis or fistulas should be resected. Strictureplasty should be performed in patients with short fibrotic stenosis in the small intestine or in those with a short length of small intestine remaining. C1 (Japan V, overseas $\mathrm{V}$; 9).

\section{Comments}

The postoperative recurrence of $\mathrm{CD}$ after resection of the intestine is unrelated to the distance between the lesion and the resected end [162], or to the histological residue of the lesion at the resected ends [163]. Therefore, in principle, a short segment should be resected. In patients with an intestinal fistula induced by another intestinal lesion, the 
intestinal area of the principal lesion is resected, and the fistulized area is wedge-resected. Strictureplasty is performed to preserve the small intestine. Bypass operations result in a high incidence of malignant tumors in the diverted residual lesion, and a high rate of reoperation. In principle, therefore, only gastrojejunostomy for duodenal stenosis is performed in patients with $\mathrm{CD}$.

\section{VII-3. Stenosis}

CQ4: What kind of surgery is used to treat stenosis? [164-166]

- Only the area of the lesion causing the stenosis should be resected. Strictureplasty should be performed for a short segment of fibrotic stenosis in the small intestine or in patients with a short length of small intestine remaining. B (Japan V, overseas IVb; 8).

\section{Comments}

It is a general opinion that there is little difference in postoperative recurrence rates between strictureplasty and intestinal resection [164]. In patients with a stenosis in a short segment, the Heineke-Mikulicz technique is applied; in those with stenosis over a long segment, the Finney technique or the Jaboulay technique is applied; and in those with stenoses close to one another, a more complex anastomosis technique is applied $[165,166]$. Furthermore, it is important to conduct a biopsy examination at the stenosis to exclude cancer. The effectiveness of strictureplasty on colonic stenosis is yet to be confirmed.

\section{VII-4. Perianal lesions}

CQ5: What kind of surgery is used to treat perianal lesions? [38, 167, 168]

- Perianal lesions in CD are classified as primary lesions (cavitating ulcerative lesions due to $\mathrm{CD}$ ), secondary refractory lesions (secondary lesions originating from a primary lesion via infection and other causes), and incidental lesions (lesions not associated with CD). C1 (overseas VI; 7).

- For incidental lesions, use ordinary treatments generally appropriate for such lesions. C1 (Japan VI, overseas VI; 7).

- Among the secondary refractory lesions, a Seton technique is used for low intersphincteric fistulas and ischiorectal fistulas. Consider creating a stoma in cases not responsive to the Seton technique or in those with fibrotic stenosis. C1 (Japan VI, overseas VI; 8).

\section{Comments}

In the diagnosis of perianal lesions in $\mathrm{CD}$, determine whether the lesion is a secondary refractory lesion or an incidental lesion, on the basis of the presence or absence of a primary lesion characteristic of $\mathrm{CD}$ in the anal canal and the fistula (e.g., whether or not there are multiple fistulas, the location, and so on [167]; refer to the Atlas of findings by visual observation of lesions in the anus of Crohn's disease [38]). The refractory fistulas most frequent in $\mathrm{CD}$ are secondary lesions. Cancer complications are found more commonly in the rectum and anal canal (including fistulas) in patients with long-term progression of CD [168]. For the treatment of refractory fistulas, use medical treatment (nutritional therapy, and drug therapies such as metronidazole or steroids) for the primary lesion, and try to induce remission if there are active rectal lesions. Subsequently, if no improvement is observed, use surgical treatment on the fistulas. For local treatment, as a general rule, use a Seton drainage technique to establish a drain to eventually discharge pus in the fistula. Consider creating a stoma in patients with fistulas or rectal stenosis that cannot be controlled with the Seton technique. For lesions of rectal stenosis with fistulas, consider rectal amputation.

CQ6: Is it possible to close a stoma later? [169, 170]

- In principle, a stoma that has been created because of rectal/anal lesions in $\mathrm{CD}$ is not closed because the lesions tend to recur frequently if it is closed. C1 (Japan $\mathrm{V}$, overseas $\mathrm{V} ; 7$ ).

\section{Comments}

In a report of stoma closure surgery in 16 patients with symptomatic improvement out of 42 patients who underwent colostomies for refractory rectoanal lesions as complications of $\mathrm{CD}, 75 \%$ required re-creation of the stoma [169]. It has been reported overseas that the cumulative rate of stoma closure in 5 years was $40 \%$ [170].

\section{VII-5. Postoperative management}

CQ7: What is the relapse rate after surgical treatment? [171-173]

- Relapse after intestinal resection is frequently discovered early by means of endoscopic exploration. The cumulative reoperation rates were $16-43 \%$ at 5 years and 26-65\% at 10 years. C1 (Japan V, overseas V; 8). 


\section{Comments}

Postoperative relapse has been defined separately on the basis of endoscopic or contrast imaging findings, or on the basis of reoperation, and so the reported rates of relapse vary. The rate of relapse found by means of endoscopic exploration (in an ileocolonic anastomosis) seemed to be $72 \%$ within 1 year after the operation [171], indicating that the lesions tend to recur early. It was reported that the cumulative reoperation rates were $16-43 \%$ at 5 years and $26-65 \%$ at 10 years [172, $173]$.

CQ8: What are the risk factors for a relapse? [164, 172, 174-176]

- Gender and the presence of granuloma are not significant risk factors. In patients who have had operations on the colonic lesions of $\mathrm{CD}$, reoperation is not frequent. C1 (overseas V; 7).

- The length of the uninvolved area in the resection margins is not a significant risk factor. $\mathrm{C} 1$ (overseas $\mathrm{V}$; 7).

- No consensus has been reached on the disease duration before the initial operation, the presence or absence of histological inflammation at the resection margins, or the types of anastomosis (end-to-end, end-to-side, or functional end-to-end) as risk factors. C1 (Japan VI, overseas VI; 8).

- In patients where fistulization is the surgical indication, the reoperation rate may be higher in comparison with that in patients with a non-fistulizing type. B (overseas $\mathrm{IVb} ; 8)$.

- It was reported that there was little difference in terms of recurrence rates between strictureplasty and intestinal resection. C1 (overseas IVb: 7).

\section{Comments}

Risk factors for recurrence have not been determined. Gender and the presence or absence of granuloma are not significant risk factors. In patients who have had operations on the colonic lesions of $\mathrm{CD}$, reoperation is not frequent [172]. The length of the intact area in the resection margins is not a significant risk factor [174]. There are contradictory reports on the disease duration before the initial operation and the presence or absence of histological inflammation at the resection margins as risk factors. There is no consensus on the type of anastomosis (end-to-end, end-to-side, or functional end-to-end) as a risk factor. Reports about the types of surgical indication (perforating versus non-perforating) are also contradictory [175]; however, a meta-analysis has indicated that the perforating type shows a higher reoperation rate [176]. It was reported that there was little difference in terms of recurrence rates between strictureplasty and intestinal resection [164].

CQ9: How can postoperative relapse be prevented?

[105, 177-179]

- There are no established measures to prevent relapse. C1 (Japan VI, overseas VI; 7).

- 5-ASA, 6-MP*, and metronidazole* may be effective in preventing a postoperative relapse. B (overseas II; 7). * Not covered by Japanese public health insurance for treatment of CD.

- The effect of postoperative nutritional therapies to prevent relapse is unclear. C1 (Japan V; 7).

\section{Comments}

The effects of surgical procedures, drug therapies, and nutritional therapies on relapse have been studied. With regard to surgical procedures, there are different views on the comparison between conventional end-to-end anastomosis and functional end-to-end anastomosis (in which the anastomotic site opening is wider in order to improve the retention of intestinal contents, which is considered to be a cause of relapse).

5-ASA (3,000 mg daily) exhibited significantly better results, in terms of clinical symptoms and relapse rate defined by endoscopy and contrast radiography, in comparison with a placebo group [177]. In patients who underwent ileal resection, metronidazole $(20 \mathrm{mg} / \mathrm{kg})$ was superior to placebo in terms of endoscopic relapse at 3 months after the operation, and in regard to the relapse rate at 1 year, but the relapse rates in the two groups were similar at 2 and 3 years after the operation [178]. In subjects with ileal resection, the 6-MP group (50 mg daily) exhibited significantly lower recurrence rates, according to the clinical symptoms, and endoscopic and contrast radiographic findings, at 2 years after the operation in comparison with the 5-ASA group and the placebo group [179]. Corticosteroids do not have efficacy to prevent postoperative relapse. Studies of the effects of anti-TNF agents to prevent postoperative recurrence have not been sufficient to reach definitive conclusions on such effects.

The long-term application of nutritional therapy (approximately 1,000 kcal daily) is difficult in many cases. A report has indicated that nutritional therapies were effective in preventing postoperative relapse [105], but the issue remains controversial. 


\section{Follow-up}

\section{VIII-1 Routine follow-up schedule}

CQ1: How are CD patients followed, and what kind of examinations are required? $[180,181]$

- Advise patients to have regular examinations, and observe changes in the clinical symptoms (abdominal pain, diarrhea, fever, and others). C1 (Japan VI, overseas VI; 8).

- CRP, ESR, complete blood counts, and serum albumin level correlate with the disease activity. C1 (Japan VI, overseas VI; 8).

- If changes in disease activity are noted, employ imaging examinations to observe the lesions. C1 (Japan VI, overseas VI; 8).

\section{Comments}

Blood tests are convenient and are the first-line examination to observe progress. CRP and ESR in particular were reported to correlate with the disease activity. Anemia and hypoalbuminemia can be indices of broadly spread lesions or highly active lesions, and hypoproteinemia is often found, particularly in patients with smallintestinal lesions. When changes in the disease activity are noted (clinical relapse, bowel obstruction, abscess, fistula), it is advisable to conduct examinations according to the previous pathophysiological conditions (the disease extent and possibility of complications) [181]. To determine the activity of intra-abdominal inflammation and the extent of active lesions, abdominal and pelvic CT and MRI are useful.

To evaluate diffuse small-intestinal lesions, radiographic examinations are often more advantageous than endoscopy or capsule endoscopy [180].

\section{VIII-2. Morphological examination}

CQ2: When is endoscopy or contrast radiography necessary? [150]

- When changes in disease activity or pathophysiological conditions are noted (clinical relapse, or complications such as bowel obstruction, abscesses, and fistulas), it is advisable to perform diagnostic imaging such as endoscopy and contrast radiography to assess the disease. C1 (Japan VI, overseas VI; 8).

\section{Comments}

A study of the long-term prognosis of $\mathrm{CD}$ indicates that even in patients with the non-stricture and non-penetrating type without complications in their initial stage, approximately $30 \%$ progressed to having stenosis or fistulas [150]. It is not unlikely that the lesions progress even in patients with stable disease, and therefore, annual endoscopic examinations or contrast radiographic examinations, as far as possible, would be helpful in assessing the pathological conditions.

\section{VIII-3. Cancer surveillance}

CQ3: Is the risk of cancer increased in $\mathrm{CD}$, and can it be prevented? [25, 26, 182-185]

- Both colonic and ileocolonic $\mathrm{CD}$ have a higher risk of colorectal and/or anal cancer than that in the general population. B (Japan IVb, overseas IVa: 8).

- The incidence of small-intestinal cancer in patients with $\mathrm{CD}$ is low, but the relative risk is high in such patients. C1 (overseas IVa; 7).

- There is no evidence that the administration of immunomodulators increases the incidence of malignant tumors. C1 (overseas IVa; 7).

- Preventive measures against the occurrence of cancer in patients with $\mathrm{CD}$ are not known, but control of the intestinal inflammation is considered to be important. C1 (Japan VI, overseas VI: 7).

- There are no data to clearly show that 5-ASA reduces the risk of colorectal cancer in CD. C1 (overseas VI; 7).

\section{Comments}

A meta-analysis of analytical epidemiological studies indicated that the relative risk of colorectal cancer was 2.5 in all types of $\mathrm{CD}$, and significantly higher, at 4.5 , in colonic-type $\mathrm{CD}$; the relative risk of small-intestinal cancer was extremely high, at 33.2, in all types of CD [182]. Another epidemiological study indicated similar results [183]. Although Japanese data on these risks are sparse, some studies of the colorectal/anal canal cancer and smallintestinal cancer complications of CD indicate that most cases are found as advanced cancer, but the incidence is not different from that in Europe and the United States [25, 26].

A meta-analysis of analytical epidemiological studies has reported that there was no significant difference in the 
occurrence of malignant tumors between groups with and without administration of immunomodulators [184]. The TREAT study presents no evidence that a group in which infliximab was administered had an increased occurrence of malignant tumors. However, it has been reported that 13 cases of hepato-splenic T-cell lymphoma (HSTCL) occurred in a group in which infliximab and AZA were administered in combination [185]. No causal relationship has been established between such drugs and cancer.

It is assumed that controlling the intestinal inflammation is effective in preventing cancer in CD, as it is in UC. It has been suggested that 5-ASA has a suppressive effect on inflammatory carcinogenesis in UC, but there are no such data for CD.

CQ4: How is cancer surveillance conducted? $[25,186]$

- There is no effective cancer surveillance program at present. C1 (Japan VI, overseas VI; 8).

\section{Comments}

In patients with long-term disease, it is advisable to conduct endoscopic and contrast imaging examinations, as appropriate, as well as to check the condition of anal fistulas. However, there are patients in whom it is difficult to conduct examinations to evaluate the small intestine in detail in the presence of stenosis. Appropriate determination of a high-risk group and an effective screening program are awaited.

In observing the progression of $\mathrm{CD}$, it should be noted that the risks of small-intestinal cancer and colorectal cancer are high, particularly in the long-term progression of the disease, and that anal fistula cancer with diagnostic difficulty does occur, although the frequency is unknown. It was reported that surveillance endoscopy, like that for UC, was helpful in finding colorectal cancer [25, 186]. However, several issues remain unsolved, such as how to conduct surveillance in patients with stenotic lesions, and therefore surveillance colonoscopy has not yet become a general practice.

CQ5: Does CD increase the risk of extra-intestinal malignant tumors, and how is surveillance for such tumors conducted? [185, 187, 188]

- A combination of infliximab and immunomodulators may increase the risk of malignant lymphoma. C1 (overseas V; 7).

- There is no established program for the surveillance of malignant tumors in regions other than the intestinal tract. C1 (Japan VI, overseas VI; 8).

\section{Comments}

In patients with rheumatoid arthritis (RA), a study reported that it was unlikely that a combination of an anti-TNF agent with MTX raised the risk of lymphoma; on the other hand, another study indicated that the long-term use of a combination of an anti-TNF agent with an immunomodulator (AZA or 6-MP) did not raise the risk of solid cancer, but did raise the risk of malignant lymphoma [187, 188]. In particular, it has been reported that HSTCL, which is extremely rare, occurred in $\mathrm{CD}$ groups receiving combination therapy [185]. The pathophysiology of CD is different from that of RA, and the concomitant therapeutic drugs used and the clinical courses are different; because of these problems, no conclusion has been reached in regard to the risk of lymphoma with combination therapy in $\mathrm{CD}$.

\section{Pregnancy and delivery}

\section{IX-1. Pregnancy}

CQ1: Is CD exacerbated during pregnancy or in relation to the menstrual cycle? [189]

- There is no evidence that $\mathrm{CD}$ is exacerbated by either pregnancy or the menstrual cycle. C1 (Japan VI, overseas $\mathrm{V} ; 8)$.

\section{Comments}

Not many studies have been carried out to evaluate the effect of pregnancy on $\mathrm{CD}$. A study involving a small number of pregnant $\mathrm{CD}$ patients (12 patients; 18 pregnancies) indicated that pregnancy was unlikely to be a factor exacerbating $\mathrm{CD}$ [189]. Adherence with taking medications, however, may get worse due to the fear of taking drugs during pregnancy. It is necessary to explain the need for the medications to pregnant patients, so that the patients have a good understanding of the benefits and harms of the drugs.

There are no reports on the relationship between the menstrual cycle and exacerbation of $\mathrm{CD}$, and clinical experience indicates that the cycle has no significant effect on $\mathrm{CD}$. However, there is a possibility that variations in estrogen secretion have some effect on the immune system, and this requires further study.

CQ2: Do CD patients have different fertility rates from those of healthy individuals? [190-193]

- Many reports indicate that patients with $\mathrm{CD}$ have reduced fertility; on the other hand, some other reports 
show no significant differences from fertility rates in the general population. B (overseas IVb; 8).

\section{Comments}

Many reports have indicated that both male and female patients with $\mathrm{CD}$ have fewer children than the general population [190-192]. It was reported that the frequency of sexual intercourse was lower in female patients with $C D$ because they were afraid of abdominal pain or fecal leaks [193]. There is a possibility that men who are administered SASP have reduced fertility [191].

CQ3: Is modification of the treatment necessary for pregnant patients with CD? [194-202]

- Devise treatment strategies according to the disease activity, considering the benefits and harms of the drugs. C1 (Japan VI, overseas VI; 8).

- Dominant overseas opinions are to treat pregnant CD patients similarly to non-pregnant patients. C1 (overseas VI; 8).

- In Japan, 5-ASA preparations, small to medium doses of steroids, and nutritional therapies are considered to be relatively safe in pregnant patients, but it is desirable to avoid immunomodulators. C1 (Japan VI; 7).

- When using nutritional therapies in pregnant patients, avoid excessive administration of vitamin A. C1 (Japan VI, overseas VI; 8).

- There is a possibility that the administration of AZA or 6-MP is associated with pre-term delivery, low birth weight, and fetal malformation. C1 (overseas IVa; 7).

- Infliximab has been reported to be relatively safe, but the relevant data are not sufficient. B (overseas IVb; 8).

\section{Comments}

Experts in Japan and overseas have different opinions concerning drug therapies for pregnant patients. Japanese specialists are cautious about using drugs because of the possibility of adverse effects, while overseas specialists assign priority to the benefits of the drugs unless they are confirmed to be harmful.

Several reports have indicated that the relative risks of pre-term delivery, low birth weight, and fetal deformation were high in groups administered AZA and 6-MP [194]; however, this does not exclude the possibility that other factors, such as high CD disease activity, were involved in these patients. Moreover, CD itself could pose such risks [195-197]. Recently, the number of reports overseas that have emphasized the safety of AZA has increased [198, 199].
Some reports have emphasized that the administration of infliximab or adalimumab in pregnancy was not associated with abnormal births; however, these reports were based on studies with a small number of subjects, and thus the safety of these drugs in pregnant patients could not be guaranteed [200, 201]. It has been reported that 5-ASA preparations are comparatively safe in pregnancy [202].

CQ4: What is the treatment for CD exacerbation during pregnancy? [198, 199, 201-203]

- Devise treatment strategies according to the disease condition, considering the benefits and harms of the drugs. C1 (Japan VI, overseas VI; 8).

- First increase the dose of a 5-ASA preparation, and reinforce nutritional therapy. C1 (Japan VI; 8).

- If the result of the above is not sufficient, use steroids, an immunomodulator, and/or an anti-TNF agent, considering their benefits and harms. C1 (Japan VI; 7).

\section{Comments}

According to the FDA's pharmaceutical categories for safety in pregnancy, 5-ASA preparations and infliximab are in category $\mathrm{B}$, oral prednisolone is in category $\mathrm{C}$, and AZA and 6-MP are in category D [203]. A study of 131 pregnant women (with RA or CD) who were treated with infliximab indicated that $15 \%$ experienced birth abnormalities, and therapeutic abortion was induced in $19 \%$; these findings correspond to the expected values for the general American population [201].

Despite the information mentioned above, many overseas textbooks recommend that the risk of such drugs be compared with the risk of CD relapse if their administration is terminated. Specifically, many overseas opinions appear to consider the risk of relapse or exacerbation of $\mathrm{CD}$ to pose a greater risk to pregnancy. As a result, many consider that $\mathrm{AZA}$ and 6-MP are relatively safe, and that they should continue to be administered when the patient becomes pregnant [198, 199]. On the other hand, MTX is contraindicated for pregnant women [202].

\section{IX-2. Lactation}

CQ5. What is the treatment for CD during a period of lactation? [202, 204, 205]

- Only a few drugs have been proven to be safe during breastfeeding, but nutritional therapy is considered safe. C1 (Japan VI, overseas VI; 8). 
- Devise treatment strategies according to the disease activity, considering the benefits and harms. C1 (Japan VI, overseas VI; 8).

\section{Comments}

Data are sparse on the transfer of therapeutic drugs for CD into breast milk and potential exposure to breastfed infants. It has been found that 5-ASA is transferred into breast milk [204, 205]. However, there are many opinions that suggest it is safe in normal use. It is advisable to avoid administering a high dose of 5-ASA to lactating women. There are no relevant data with respect to AZA and 6-MP; on the other hand, MTX and cyclosporine are contraindicated in lactating women [202].

Acknowledgments This study was supported by the Health and Labour Sciences Research Grants for Research on Intractable Diseases from the Ministry of Health, Labour and Welfare of Japan.

Conflict of interest Toshiyuki Matsui received research grants from Eisai Co., Ltd., Mitsubishi Tanabe Pharma Co., Ajinomoto Pharmaceuticals Co., Ltd., Zeria Pharmaceutical Co., Ltd., Kyorin Pharmaceutical Co., Ltd., JIMRO Co., Ltd., Astellas Pharma Inc.; received lecture fees from Eisai Co., Ltd., ABBOTT JAPAN Co., Ltd. Mamoru Watanabe received research grants from ABBOTT JAPAN Co., Ltd, Astellas Pharma Inc., Asahi Kasei Kuraray Medical Co., Ltd, Ajinomoto Pharmaceuticals Co., Ltd, AstraZeneca, Chugai Pharmaceutical Co., Ltd, DAIICHI SANKYO CO., Ltd., Eisai Co., Ltd, Kyowa Hakko Kirin Co., Ltd, Kyorin Pharmaceutical Co. Ltd, JIMRO Co., Ltd, Mitsubishi Tanabe Pharma Co., MSD K.K., Otsuka Pharma Co., Ltd, UCB Japan Co., Ltd, and Zeria Pharmaceutical Co., Ltd. Toshifumi Hibi serves as a consultant to Mitsubishi Tanabe Pharma Co.; Toshifumi Hibi received research grants from Abbott Japan Co., Ltd., Takeda Pharmaceutical Co., Ltd., Ajinomoto Pharmaceuticals Co., Ltd., Zeria Pharmaceutical Co., Ltd., Mitsubishi Tanabe Pharma Co., Asahi Kasei Kuraray Medical Co., Ltd., and AstraZeneca. Fumiaki Ueno and Katsuyoshi Matsuoka have no conflict of interest.

Open Access This article is distributed under the terms of the Creative Commons Attribution Noncommercial License which permits any noncommercial use, distribution, and reproduction in any medium, provided the original author(s) and the source are credited.

\section{References}

1. Shiffman RN, Shekelle P, Overhage JM, Slutsky J, Grimshaw J, Deshpande AM. Standardized reporting of clinical practice guidelines: a proposal from the Conference on Guideline Standardization. Ann Intern Med. 2003;139:493-8.

2. Jewell DP. Oxford textbook of medicine. 4th ed. Oxford University Press, Oxford; 2005.

3. Morita N, Toki S, Hirohashi T, Minoda T, Ogawa K, Kono S, et al. Incidence and prevalence of inflammatory bowel disease in Japan: nationwide epidemiological survey during the year 1991 . J Gastroenterol. 1995;30:1-4.

4. Yao T, Matsui T, Hiwatashi N. Crohn's disease in Japan: diagnostic criteria and epidemiology. Dis Colon Rectum. 2000;43:S85-93.

5. Ohta A, Nagai M, Nishina M, Shibazaki S, Ishijima H, Izumida $\mathrm{M}$. Age at the onset of intractable disease: based on a clinical database for patients receiving financial aid for treatment. Nihon Koshu Eisei Zasshi. 2007;54:3-14 (in Japanese).

6. Loftus EV Jr. Clinical epidemiology of inflammatory bowel disease: incidence, prevalence, and environmental influences. Gastroenterology. 2004;126:1504-17.

7. Loftus EV Jr, Schoenfeld P, Sandborn WJ. The epidemiology and natural history of Crohn's disease in population-based patient cohorts from North America: a systematic review. Aliment Pharmacol Ther. 2002;16:51-60.

8. Isaacs KL, Lewis JD, Sandborn WJ, Sands BE, Targan SR. State of the art: IBD therapy and clinical trials in IBD. Inflamm Bowel Dis. 2005;11:S3-12.

9. Hibi T, Ogata H. Novel pathophysiological concepts of inflammatory bowel disease. J Gastroenterol. 2006;41:10-6.

10. Sands BE. Inflammatory bowel disease: past, present, and future. J Gastroenterol. 2007;42:16-25.

11. Orholm M, Munkholm P, Langholz E, Nielsen OH, Sorensen TI, Binder V. Familial occurrence of inflammatory bowel disease. N Engl J Med. 1991;324:84-8.

12. Peeters M, Nevens H, Baert F, Hiele M, de Meyer AM, Vlietinck R, et al. Familial aggregation in Crohn's disease: increased age-adjusted risk and concordance in clinical characteristics. Gastroenterology. 1996;111:597-603.

13. Jarnerot G, Jarnmark I, Nilsson K. Consumption of refined sugar by patients with Crohn's disease, ulcerative colitis, or irritable bowel syndrome. Scand J Gastroenterol. 1983;18:999-1002.

14. Tragnone A, Valpiani D, Miglio F, Elmi G, Bazzocchi G, Pipitone E, et al. Dietary habits as risk factors for inflammatory bowel disease. Eur J Gastroenterol Hepatol. 1995;7:47-51.

15. Sakamoto N, Kono S, Wakai K, Fukuda Y, Satomi M, Shimoyama $\mathrm{T}$, et al. Dietary risk factors for inflammatory bowel disease: a multicenter case-control study in Japan. Inflamm Bowel Dis. 2005;11:154-63.

16. Ryan WR, Allan RN, Yamamoto T, Keighley MR. Crohn's disease patients who quit smoking have a reduced risk of reoperation for recurrence. Am J Surg. 2004;187:219-25.

17. Parsi MA, Achkar JP, Richardson S, Katz J, Hammel JP, Lashner BA, et al. Predictors of response to infliximab in patients with Crohn's disease. Gastroenterology. 2002;123:707-13.

18. Felder JB, Korelitz BI, Rajapakse R, Schwarz S, Horatagis AP, Gleim G. Effects of nonsteroidal antiinflammatory drugs on inflammatory bowel disease: a case-control study. Am J Gastroenterol. 2000;95:1949-54.

19. Boyko EJ, Theis MK, Vaughan TL, Nicol-Blades B. Increased risk of inflammatory bowel disease associated with oral contraceptive use. Am J Epidemiol. 1994;140:268-78.

20. Gasche C, Scholmerich J, Brynskov J, D'Haens G, Hanauer SB, Irvine EJ, et al. A simple classification of Crohn's disease: report of the Working Party for the World Congresses of Gastroenterology, Vienna 1998. Inflamm Bowel Dis. 2000;6: $8-15$.

21. Carter MJ, Lobo AJ, Travis SP. Guidelines for the management of inflammatory bowel disease in adults. Gut. 2004;53: V1-16.

22. Ueno F, Ide H, Yanagawa T. Factors that affect the status of employment of patients with Crohn's disease: a study of subjective and objective indexes. Annual reports of research group on QOL in specified disease cases supported by the Ministry of Health, Labour, and Welfare of Japan, Tokyo, Japan, 1998. p. 125-36 (in Japanese).

23. Takebayashi T, Nishiwaki Y, Asakura K, Kitabora T, Inoue N. An epidemiological study on the incidence, prevalence, and prognosis of intractable inflammatory bowel disorders. Annual reports of the research group of intractable inflammatory bowel disease subsidized by the Ministry of Health, Labour, and Welfare of Japan, Tokyo, Japan, 2007. p. 79-83 (in Japanese). 
24. von Roon AC, Reese G, Teare J, Constantinides V, Darzi AW, Tekkis PP. The risk of cancer in patients with Crohn's disease. Dis Colon Rectum. 2007;50:839-55.

25. Matsui T, Yano Y, Hirai F. Crohn's disease and intestinal cancer. Nihon Shokakibyo Gakkai Zasshi. 2006;103:797-804 (in Japanese).

26. Yano Y, Matsui T, Uno H, Hirai F, Futami K, Iwashita A. Risks and clinical features of colorectal cancer complicating Crohn's disease in Japanese patients. J Gastroenterol Hepatol. 2008;23: 1683-8.

27. Wolters FL, Russel MG, Stockbrugger RW. Systematic review: has disease outcome in Crohn's disease changed during the last four decades? Aliment Pharmacol Ther. 2004;20:483-96.

28. Oriuchi T, Hiwatashi N, Kinouchi Y, Takahashi S, Takagi S, Negoro K, et al. Clinical course and longterm prognosis of Japanese patients with Crohn's disease: predictive factors, rates of operation, and mortality. J Gastroenterol. 2003;38:942-53.

29. Uno H, Yao T, Matsui T, Sakurai T, Iida M, Matsumoto T, et al. Mortality and cause of death in Japanese patients with Crohn's disease. Dis Colon Rectum. 2003;46:S15-21.

30. Forbes A. Clinical presentation and diagnosis of Crohn's disease. In: Satsangi J, Southerland L, editors. Inflammatory bowel disease. Philadelphia: Churchill Livingstone; 2003.

31. Beaugerie L, Seksik P, Nion-Larmurier I, Gendre JP, Cosnes J. Predictors of Crohn's disease. Gastroenterology. 2006;130: 650-6.

32. Orchard TR, Thiyagaraja S, Welsh KI, Wordsworth BP, Hill Gaston JS, Jewell DP. Clinical phenotype is related to HLA genotype in the peripheral arthropathies of inflammatory bowel disease. Gastroenterology. 2000;118:274-8.

33. Munkholm P, Langholz E, Nielsen $\mathrm{OH}$, Kreiner S, Binder V. Incidence and prevalence of Crohn's disease in the county of Copenhagen, 1962-87: a sixfold increase in incidence. Scand J Gastroenterol. 1992;27:609-14.

34. Bernstein CN, Blanchard JF, Rawsthorne P, Yu N. The prevalence of extraintestinal diseases in inflammatory bowel disease: a population-based study. Am J Gastroenterol. 2001;96:1116-22.

35. Travis S, Innes N, Davies MG, Daneshmend T, Hughes S. Sweet's syndrome: an unusual cutaneous feature of Crohn's disease or ulcerative colitis. The South West Gastroenterology Group. Eur J Gastroenterol Hepatol. 1997;9:715-20.

36. Sandborn WJ, Fazio VW, Feagan BG, Hanauer SB. AGA technical review on perianal Crohn's disease. Gastroenterology. 2003;125:1508-30.

37. Hughes LE. Surgical pathology and management of anorectal Crohn's disease. J R Soc Med. 1978;71:644-51.

38. Futami K. Atlas of findings by visual observation of lesions in the anus of Crohn's disease. Annual reports of the research group of intractable inflammatory bowel disease subsidized by the Ministry of Health, Labour, and Welfare of Japan, Tokyo, Japan, 2006. Suppl. (in Japanese).

39. Matsui T, Hirai F, Hisabe T. Proposed diagnostic criteria for Crohn's disease. Annual reports of the research group of intractable inflammatory bowel disease subsidized by the Ministry of Health, Labour, and Welfare of Japan, Tokyo, Japan, 2011. p. 52-4 (in Japanese).

40. Hommes DW, van Deventer SJ. Endoscopy in inflammatory bowel diseases. Gastroenterology. 2004;126:1561-73.

41. Witte AM, Veenendaal RA, Van Hogezand RA, Verspaget HW, Lamers CB. Crohn's disease of the upper gastrointestinal tract: the value of endoscopic examination. Scand J Gastroenterol Suppl. 1998;225:100-5.

42. Best WR, Becktel JM, Singleton JW, Kern F Jr. Development of a Crohn's disease activity index. National Cooperative Crohn's Disease Study. Gastroenterology. 1976;70:439-44.
43. Mary JY, Modigliani R. Development and validation of an endoscopic index of the severity for Crohn's disease: a prospective multicentre study. Groupe d'Etudes Therapeutiques des Affections Inflammatoires du Tube Digestif (GETAID). Gut. 1989;30:983-9.

44. Denis MA, Reenaers C, Fontaine F, Belaiche J, Louis E. Assessment of endoscopic activity index and biological inflammatory markers in clinically active Crohn's disease with normal C-reactive protein serum level. Inflamm Bowel Dis. 2007;13:1100-5.

45. IBD Working Group of the European Society for Paediatric Gastroenterology Hepatology, and Nutrition. Inflammatory bowel disease in children and adolescents: recommendations for diagnosis - the Porto criteria. J Pediatr Gastroenterol Nutr. 2005;41:1-7.

46. Pera A, Bellando P, Caldera D, Ponti V, Astegiano M, Barletti $\mathrm{C}$, et al. Colonoscopy in inflammatory bowel disease. Diagnostic accuracy and proposal of an endoscopic score. Gastroenterology. 1987;92:181-5.

47. Saibeni S, Rondonotti E, Iozzelli A, Spina L, Tontini GE, Cavallaro F, et al. Imaging of the small bowel in Crohn's disease: a review of old and new techniques. World J Gastroenterol. 2007;13:3279-87.

48. Taruishi M, Saitoh Y, Watari J, Ashida T, Ayabe T, Takemura $\mathrm{K}$, et al. Balloon-occluded endoscopic retrograde ileography. Radiology. 2000;214:908-11.

49. Iida M, Aoyagi K, Atsushi K. Crohn's disease-X-ray examination of the small intestine. Stomach Intestine. 1997;32:341-50 (in Japanese).

50. Tominaga M. Clinical features of Crohn's disease: relationship of disease type and severity to clinical findings at the time of diagnosis in 166 cases. Fukuoka Igaku Zasshi. 1992;83:6-20 (in Japanese).

51. Horsthuis K, Stokkers PC, Stoker J. Detection of inflammatory bowel disease: diagnostic performance of cross-sectional imaging modalities. Abdom Imaging. 2008;33:407-16.

52. Nikolaus S, Schreiber S. Diagnostics of inflammatory bowel disease. Gastroenterology. 2007;133:1670-89.

53. Ota $\mathrm{Y}$, Matsui $\mathrm{T}$, Ono $\mathrm{H}$, Uno $\mathrm{H}$, Matake $\mathrm{H}$, Tsuda $\mathrm{S}$, et al. Value of virtual computed tomographic colonography for Crohn's colitis: comparison with endoscopy and barium enema. Abdom Imaging. 2003;28:778-83.

54. Meucci G, Bortoli A, Riccioli FA, Girelli CM, Radaelli F, Rivolta $\mathrm{R}$, et al. Frequency and clinical evolution of indeterminate colitis: a retrospective multi-centre study in northern Italy. GSMII (Gruppo di Studio per le Malattie Infiammatorie Intestinali). Eur J Gastroenterol Hepatol. 1999;11:909-13.

55. Matsui T, Yao T, Sakurai T, Yao K, Hirai F, Matake H, et al. Clinical features and pattern of indeterminate colitis: Crohn's disease with ulcerative colitis-like clinical presentation. J Gastroenterol. 2003;38:647-55.

56. Hanauer SB, Sandborn W. Management of Crohn's disease in adults. Am J Gastroenterol. 2001;96:635-43.

57. Travis SP, Stange EF, Lemann M, Oresland T, Chowers Y, Forbes A, et al. European evidence based consensus on the diagnosis and management of Crohn's disease: current management. Gut. 2006;55:116-35.

58. Iida M. A study of drug therapies for Crohn's disease: proposed revision of the clinical practice guidelines for Crohn's disease. Annual reports of the research group of intractable inflammatory bowel disease subsidized by the Ministry of Health, Labour, and Welfare of Japan, Tokyo, Japan, 2007. p. 27 (in Japanese).

59. Farrell R, Peppercorn MA. Medical management of Crohn's disease in adults. In: Basow DS, editor. UpToDate. Waltham; 2007. 
60. Hiwatashi N. Crohn's disease Q\&A. Annual reports of the research group of intractable inflammatory bowel disease subsidized by the Ministry of Health, Labour, and Welfare of Japan, Tokyo, Japan, 1999. p. 43-6 (in Japanese).

61. Hiwatashi N, Takazoe M. Proposed guidelines for the management of patients with Crohn's disease. Annual reports of the research group of intractable inflammatory bowel disease subsidized by the Ministry of Health, Labour, and Welfare of Japan, Tokyo, Japan, 2002. p. 220-3 (in Japanese).

62. Adam D, Adam J, Price H. An analysis of an inflammatory bowel disease practice in an urban community hospital. Can J Gastroenterol. 2000;14:483-8.

63. Hirai F, Takatsu N, Ninomiya K, Baba T, Kanemitsu T, Morokuma T, et al. Long-term clinical course and prognosis of patients with Crohn's disease. Stomach Intestine. 2007;42:1843-58 (in Japanese).

64. Ng V, Millard W, Lebrun C, Howard J. Exercise and Crohn's disease: speculations on potential benefits. Can J Gastroenterol. 2006;20:657-60.

65. Mahid SS, Minor KS, Soto RE, Hornung CA, Galandiuk S. Smoking and inflammatory bowel disease: a meta-analysis. Mayo Clin Proc. 2006;81:1462-71.

66. Mahid SS, Minor KS, Stromberg AJ, Galandiuk S. Active and passive smoking in childhood is related to the development of inflammatory bowel disease. Inflamm Bowel Dis. 2007;13:431-8.

67. Avidan B, Sakhnini E, Lahat A, Lang A, Koler M, Zmora O, et al. Risk factors regarding the need for a second operation in patients with Crohn's disease. Digestion. 2005;72:248-53.

68. Cosnes J, Carbonnel F, Carrat F, Beaugerie L, Cattan S, Gendre J. Effects of current and former cigarette smoking on the clinical course of Crohn's disease. Aliment Pharmacol Ther. 1999;13:1403-11.

69. Russel MG, Volovics A, Schoon EJ, van Wijlick EH, Logan RF, Shivananda $\mathrm{S}$, et al. Inflammatory bowel disease: is there any relation between smoking status and disease presentation? European Collaborative IBD Study Group. Inflamm Bowel Dis. 1998;4:182-6.

70. Cosnes J, Beaugerie L, Carbonnel F, Gendre JP. Smoking cessation and the course of Crohn's disease: an intervention study. Gastroenterology. 2001;120:1093-9.

71. Vermeire S, Louis E, Carbonez A, Van Assche G, Noman M, Belaiche J, et al. Demographic and clinical parameters influencing the short-term outcome of anti-tumor necrosis factor (infliximab) treatment in Crohn's disease. Am J Gastroenterol. 2002;97:2357-63.

72. Benchimol EI, Seow CH, Steinhart AH, Griffiths AM. Traditional corticosteroids for induction of remission in Crohn's disease. Cochrane Database Syst Rev. 2008;2:CD006792.

73. Steinhart AH, Ewe K, Griffiths AM, Modigliani R, Thomsen OO. Corticosteroids for maintenance of remission in Crohn's disease. Cochrane Database Syst Rev. 2003;4:CD000301.

74. Seow CH, Benchimol EI, Griffiths AM, Otley AR, Steinhart AH. Budesonide for induction of remission in Crohn's disease. Cochrane Database Syst Rev. 2008;3:CD000296.

75. Hanauer SB, Stromberg U. Oral Pentasa in the treatment of active Crohn's disease: a meta-analysis of double-blind, placebo-controlled trials. Clin Gastroenterol Hepatol. 2004;2: 379-88.

76. Akobeng AK, Gardener E. Oral 5-aminosalicylic acid for maintenance of medically-induced remission in Crohn's disease. Cochrane Database Syst Rev. 2005;1:CD003715.

77. Steinhart AH, Forbes A, Mills EC, Rodgers-Gray BS, Travis SP. Systematic review: the potential influence of mesalazine formulation on maintenance of remission in Crohn's disease. Aliment Pharmacol Ther. 2007;25:1389-99.

78. Sandborn W, Sutherland L, Pearson D, May G, Modigliani R, Prantera C. Azathioprine or 6-mercaptopurine for inducing remission of Crohn's disease. Cochrane Database Syst Rev. 2000;2:CD000545.

79. Pearson DC, May GR, Fick G, Sutherland LR. Azathioprine for maintaining remission of Crohn's disease. Cochrane Database Syst Rev. 2000;2:CD000067.

80. Akobeng AK, Zachos M. Tumor necrosis factor-alpha antibody for induction of remission in Crohn's disease. Cochrane Database Syst Rev. 2004;1:CD003574.

81. Behm BW, Bickston SJ. Tumor necrosis factor-alpha antibody for maintenance of remission in Crohn's disease. Cochrane Database Syst Rev. 2008;1:CD006893.

82. Sands BE, Anderson FH, Bernstein CN, Chey WY, Feagan BG, Fedorak RN, et al. Infliximab maintenance therapy for fistulizing Crohn's disease. N Engl J Med. 2004;350:876-85.

83. Hanauer SB, Sandborn WJ, Rutgeerts P, Fedorak RN, Lukas M, MacIntosh D, et al. Human anti-tumor necrosis factor monoclonal antibody (adalimumab) in Crohn's disease: the CLASSIC-I trial. Gastroenterology. 2006;130:323-33.

84. Colombel JF, Sandborn WJ, Rutgeerts P, Enns R, Hanauer SB, Panaccione R, et al. Adalimumab for maintenance of clinical response and remission in patients with Crohn's disease: the CHARM trial. Gastroenterology. 2007;132:52-65.

85. Sandborn WJ, Rutgeerts P, Enns R, Hanauer SB, Colombel JF, Panaccione R, et al. Adalimumab induction therapy for Crohn disease previously treated with infliximab: a randomized trial. Ann Intern Med. 2007;146:829-38.

86. Lichtenstein GR, Feagan BG, Cohen RD, Salzberg BA, Diamond RH, Chen DM, et al. Serious infections and mortality in association with therapies for Crohn's disease: TREAT registry. Clin Gastroenterol Hepatol. 2006;4:621-30.

87. Toruner M, Loftus EV Jr, Harmsen WS, Zinsmeister AR, Orenstein R, Sandborn WJ, et al. Risk factors for opportunistic infections in patients with inflammatory bowel disease. Gastroenterology. 2008;134:929-36.

88. Keane J, Gershon S, Wise RP, Mirabile-Levens E, Kasznica J, Schwieterman WD, et al. Tuberculosis associated with infliximab, a tumor necrosis factor alpha-neutralizing agent. N Engl J Med. 2001;345:1098-104.

89. Lichtenstein GR, Olson A, Travers S, Diamond RH, Chen DM, Pritchard ML, et al. Factors associated with the development of intestinal strictures or obstructions in patients with Crohn's disease. Am J Gastroenterol. 2006;101:1030-8.

90. Lichtenstein GR, Cohen RD, Feagan BG, Sandborn W, Salzberg BA, Chen DM, et al. Safety of infliximab and other Crohn's disease therapies - updated TREAT Registry data with over 10,000 patient-years of follow-up. Gastroenterology. 2005;128:A-580.

91. Biancone L, Orlando A, Kohn A, Colombo E, Sostegni R, Angelucci E, et al. Infliximab and newly diagnosed neoplasia in Crohn's disease: a multicentre matched pair study. Gut. 2006;55:228-33.

92. Burmester GR, Mease P, Dijkmans BA, Gordon K, Lovell D, Panaccione R, et al. Adalimumab safety and mortality rates from global clinical trials of six immune-mediated inflammatory diseases. Ann Rheum Dis. 2009;68:1863-9.

93. Rahimi R, Nikfar S, Rezaie A, Abdollahi M. A meta-analysis of broad-spectrum antibiotic therapy in patients with active Crohn's disease. Clin Ther. 2006;28:1983-8.

94. Prantera C, Kohn A, Mangiarotti R, Andreoli A, Luzi C. Antimycobacterial therapy in Crohn's disease: results of a controlled, double-blind trial with a multiple antibiotic regimen. Am J Gastroenterol. 1994;89:513-8.

95. Prantera C, Zannoni F, Scribano ML, Berto E, Andreoli A, Kohn A, et al. An antibiotic regimen for the treatment of active Crohn's disease: a randomized, controlled clinical trial of metronidazole plus ciprofloxacin. Am J Gastroenterol. 1996;91: 328-32. 
96. Steinhart AH, Feagan BG, Wong CJ, Vandervoort M, Mikolainis $\mathrm{S}$, Croitoru $\mathrm{K}$, et al. Combined budesonide and antibiotic therapy for active Crohn's disease: a randomized controlled trial. Gastroenterology. 2002;123:33-40.

97. Rutgeerts P, Van Assche G, Vermeire S, D'Haens G, Baert F, Noman M, et al. Ornidazole for prophylaxis of postoperative Crohn's disease recurrence: a randomized, double-blind, placebo-controlled trial. Gastroenterology. 2005;128:856-61.

98. Fernandez-Banares F, Cabre E, Esteve-Comas M, Gassull MA. How effective is enteral nutrition in inducing clinical remission in active Crohn's disease? A meta-analysis of the randomized clinical trials. JPEN J Parenter Enteral Nutr. 1995;19:356-64.

99. Griffiths AM, Ohlsson A, Sherman PM, Sutherland LR. Metaanalysis of enteral nutrition as a primary treatment of active Crohn's disease. Gastroenterology. 1995;108:1056-67.

100. Borrelli O, Cordischi L, Cirulli M, Paganelli M, Labalestra V, Uccini S, et al. Polymeric diet alone versus corticosteroids in the treatment of active pediatric Crohn's disease: a randomized controlled open-label trial. Clin Gastroenterol Hepatol. 2006;4: 744-53.

101. Heuschkel RB, Menache CC, Megerian JT, Baird AE. Enteral nutrition and corticosteroids in the treatment of acute Crohn's disease in children. J Pediatr Gastroenterol Nutr. 2000;31:8-15.

102. Okada M, Yao T, Yamamoto $\mathrm{T}$, Takenaka K, Imamura K, Maeda K, et al. Controlled trial comparing an elemental diet with prednisolone in the treatment of active Crohn's disease. Hepatogastroenterology. 1990;37:72-80.

103. Takagi S, Utsunomiya K, Kuriyama S, Yokoyama H, Takahashi $\mathrm{S}$, Iwabuchi M, et al. Effectiveness of an 'half elemental diet' as maintenance therapy for Crohn's disease: a randomized-controlled trial. Aliment Pharmacol Ther. 2006;24:1333-40.

104. Hirakawa H, Fukuda Y, Tanida N, Hosomi M, Shimoyama T. Home elemental enteral hyperalimentation (HEEH) for the maintenance of remission in patients with Crohn's disease. Gastroenterol Jpn. 1993;28:379-84.

105. Esaki M, Matsumoto T, Hizawa K, Nakamura S, Jo Y, Mibu R, et al. Preventive effect of nutritional therapy against postoperative recurrence of Crohn disease, with reference to findings determined by intra-operative enteroscopy. Scand J Gastroenterol. 2005;40:1431-7.

106. Sakurai T, Matsui T, Yao T, Takagi Y, Hirai F, Aoyagi K, et al. Short-term efficacy of enteral nutrition in the treatment of active Crohn's disease: a randomized, controlled trial comparing nutrient formulas. JPEN J Parenter Enteral Nutr. 2002;26:98103.

107. Kobayashi K, Katsumata T, Yokoyama K, Takahashi H, Igarashi M, Saigenji K. A randomized controlled study of total parenteral nutrition and enteral nutrition by elemental and polymeric diet as primary therapy in active phase of Crohn's disease. Nihon Shokakibyo Gakkai Zasshi. 1998;95:1212-21 (in Japanese).

108. Jones VA. Comparison of total parenteral nutrition and elemental diet in induction of remission of Crohn's disease. Longterm maintenance of remission by personalized food exclusion diets. Dig Dis Sci. 1987;32:100S-7S.

109. Fuchigami T, Ohgushi $H$, Imamura $K$, Yao $T$, Omae $T$, Watanabe $\mathrm{H}$, et al. Effects of total parenteral nutrition on colonic lesions in Crohn's disease: radiographic and endoscopic study. Gastroenterol Jpn. 1982;17:521-9.

110. Galandiuk S, O’Neill M, McDonald P, Fazio VW, Steiger E. A century of home parenteral nutrition for Crohn's disease. Am J Surg. 1990;159:540-4.

111. Fukuda Y, Matsui T, Suzuki Y, Kanke K, Matsumoto T, Takazoe M, et al. Adsorptive granulocyte and monocyte apheresis for refractory Crohn's disease: an open multicenter prospective study. J Gastroenterol. 2004;39:1158-64.
112. Thirlby RC, Land JC, Fenster LF, Lonborg R. Effect of surgery on health-related quality of life in patients with inflammatory bowel disease: a prospective study. Arch Surg. 1998;133: 826-32.

113. Homer DR, Grand RJ, Colodny AH. Growth, course, and prognosis after surgery for Crohn's disease in children and adolescents. Pediatrics. 1977;59:717-25.

114. Fukushima T, Sugita A. A study of factors for postoperative recurrence of Crohn's disease. Annual reports of the research group of intractable inflammatory bowel disease subsidized by the Ministry of Health, Labour, and Welfare of Japan, Tokyo, Japan, 1995. p. 99-102 (in Japanese).

115. Matsui T, Tsuda S, Matake H, Ikeda K, Yao T. Long-term outcome of endoscopic balloon dilation in obstructive gastrointestinal Crohn's disease. Dig Endosc. 2004;16:S27-30.

116. Hassan C, Zullo A, De Francesco V, Ierardi E, Giustini M, Pitidis A, et al. Systematic review: endoscopic dilatation in Crohn's disease. Aliment Pharmacol Ther. 2007;26:1457-64.

117. Anthonisen P, Barany F, Folkenborg O, Holtz A, Jarnum S, Kristensen M, et al. The clinical effect of salazosulphapyridine (Salazopyrin) in Crohn's disease. A controlled double-blind study. Scand J Gastroenterol. 1974;9:549-54.

118. Rijk MC, van Hogezand RA, van Lier HJ, van Tongeren JH. Sulphasalazine and prednisone compared with sulphasalazine for treating active Crohn disease. A double-blind, randomized, multicenter trial. Ann Intern Med. 1991;114:445-50.

119. Summers RW, Switz DM, Sessions JT Jr, Becktel JM, Best WR, Kern F Jr, et al. National Cooperative Crohn's Disease Study: results of drug treatment. Gastroenterology. 1979;77:847-69.

120. Munakata A, Hiwatashi N, Muto T, Tsuchiya S, Fukushima T, Kobayashi J, et al. Clinical effects of N-5ASA (oral controlled -release mesalazine) on Crohn's disease: a multicenter, openlabel, dose-ranging study). Jpn Pharmacol Ther. 1994;22: S2531-54 (in Japanese).

121. Singleton JW, Hanauer SB, Gitnick GL, Peppercorn MA, Robinson MG, Wruble LD, et al. Mesalamine capsules for the treatment of active Crohn's disease: results of a 16-week trial. Pentasa Crohn's Disease Study Group. Gastroenterology. 1993;104:1293-301.

122. Prantera C, Cottone M, Pallone F, Annese V, Franze A, Cerutti $\mathrm{R}$, et al. Mesalamine in the treatment of mild to moderate active Crohn's ileitis: results of a randomized, multicenter trial. Gastroenterology. 1999;116:521-6.

123. Colombel JF, Lemann M, Cassagnou M, Bouhnik Y, Duclos B, Dupas JL, et al. A controlled trial comparing ciprofloxacin with mesalazine for the treatment of active Crohn's disease. Groupe d'Etudes Therapeutiques des Affections Inflammatoires Digestives (GETAID). Am J Gastroenterol. 1999;94:674-8.

124. Zoli G, Care M, Parazza M, Spano C, Biagi PL, Bernardi M, et al. A randomized controlled study comparing elemental diet and steroid treatment in Crohn's disease. Aliment Pharmacol Ther. 1997; 11:735-40.

125. Feagan BG, Rochon J, Fedorak RN, Irvine EJ, Wild G, Sutherland L, et al. Methotrexate for the treatment of Crohn's disease. The North American Crohn's Study Group Investigators. N Engl J Med. 1995;332:292-7.

126. Alfadhli AA, McDonald JW, Feagan BG. Methotrexate for induction of remission in refractory Crohn's disease. Cochrane Database Syst Rev. 2005;1:CD003459.

127. Targan SR, Hanauer SB, van Deventer SJ, Mayer L, Present DH, Braakman T, et al. A short-term study of chimeric monoclonal antibody cA2 to tumor necrosis factor alpha for Crohn's disease. Crohn's Disease cA2 Study Group. N Engl J Med. 1997;337: 1029-35.

128. Hanauer SB, Feagan BG, Lichtenstein GR, Mayer LF, Schreiber $\mathrm{S}$, Colombel JF, et al. Maintenance infliximab for Crohn's 
disease: the ACCENT I randomised trial. Lancet. 2002;359: 1541-9.

129. Shaffer JA, Williams SE, Turnberg LA, Houston JB, Rowland M. Absorption of prednisolone in patients with Crohn's disease. Gut. 1983;24:182-6.

130. Whiteford MH, Kilkenny J 3rd, Hyman N, Buie WD, Cohen J, Orsay C, et al. Practice parameters for the treatment of perianal abscess and fistula-in-ano (revised). Dis Colon Rectum. 2005;48:1337-42.

131. Sandborn W, Fazio VW, Feagan BG, Hanauer SB, Committee AGACP. American Gastroenterological Association medical position statement: perianal Crohn's disease. Gastroenterology. 2003;125:1503-7.

132. Pearson DC, May GR, Fick GH, Sutherland LR. Azathioprine and 6-mercaptopurine in Crohn disease. A meta-analysis. Ann Intern Med. 1995;123:132-42.

133. Strong SA, Koltun WA, Hyman NH, Buie WD. Practice parameters for the surgical management of Crohn's disease. Dis Colon Rectum. 2007;50:1735-46.

134. Judge TA, Lichtenstein GR. Fistulizing Crohn's disease. In: Sartor RB, Sandborn W, editors. Kirsner's inflammatory bowel diseases. 6th ed. Edinburgh: Saunders; 2004. p. 700-16.

135. Vader JP, Froehlich F, Juillerat P, Burnand B, Felley C, Gonvers JJ, et al. Appropriate treatment for Crohn's disease: methodology and summary results of a multidisciplinary international expert panel approach-EPACT. Digestion. 2006;73:237-48.

136. Thomas-Gibson S, Brooker JC, Hayward CM, Shah SG, Williams CB, Saunders BP. Colonoscopic balloon dilation of Crohn's strictures: a review of long-term outcomes. Eur $\mathbf{J}$ Gastroenterol Hepatol. 2003;15:485-8.

137. Homan WP, Tang CK, Thorbjarnarson B. Acute massive hemorrhage from intestinal Crohn disease. Report of seven cases and review of the literature. Arch Surg. 1976;111:901-5.

138. Tsujikawa T, Nezu R, Andoh A, Saotome T, Araki Y, Ishizuka $\mathrm{Y}$, et al. Infliximab as a possible treatment for the hemorrhagic type of Crohn's disease. J Gastroenterol. 2004;39:284-7.

139. Robert JR, Sachar DB, Greenstein AJ. Severe gastrointestinal hemorrhage in Crohn's disease. Ann Surg. 1991;213:207-11.

140. Belaiche J, Louis E, D'Haens G, Cabooter M, Naegels S, De Vos M, et al. Acute lower gastrointestinal bleeding in Crohn's disease: characteristics of a unique series of 34 patients. Belgian IBD Research Group. Am J Gastroenterol. 1999;94:2177-81.

141. Yamaguchi A, Matsui T, Sakurai T, Ueki T, Nakabayashi S, Yao T, et al. The clinical characteristics and outcome of intraabdominal abscess in Crohn's disease. J Gastroenterol. 2004; 39:441-8.

142. Potthast S, Rieber A, Von Tirpitz C, Wruk D, Adler G, Brambs HJ. Ultrasound and magnetic resonance imaging in Crohn's disease: a comparison. Eur Radiol. 2002;12:1416-22.

143. Gervais DA, Hahn PF, O'Neill MJ, Mueller PR. Percutaneous abscess drainage in Crohn disease: technical success and shortand long-term outcomes during 14 years. Radiology. 2002;222: 645-51.

144. Gutierrez A, Lee H, Sands BE. Outcome of surgical versus percutaneous drainage of abdominal and pelvic abscesses in Crohn's disease. Am J Gastroenterol. 2006;101:2283-9.

145. Van den Bosch F, Kruithof E, De Vos M, De Keyser F, Mielants H. Crohn's disease associated with spondyloarthropathy: effect of TNF-alpha blockade with infliximab on articular symptoms. Lancet. 2000;356:1821-2.

146. Brooklyn TN, Dunnill MG, Shetty A, Bowden JJ, Williams JD, Griffiths CE, et al. Infliximab for the treatment of pyoderma gangrenosum: a randomised, double blind, placebo controlled trial. Gut. 2006;55:505-9.

147. Braun J, Brandt J, Listing J, Zink A, Alten R, Golder W, et al. Treatment of active ankylosing spondylitis with infliximab: a randomised controlled multicentre trial. Lancet. 2002;359: 1187-93.

148. Sutherland LR, Ramcharan S, Bryant H, Fick G. Effect of cigarette smoking on recurrence of Crohn's disease. Gastroenterology. 1990;98:1123-8.

149. Mawdsley JE, Rampton DS. Psychological stress in IBD: new insights into pathogenic and therapeutic implications. Gut. 2005;54:1481-91.

150. Cosnes J, Cattan S, Blain A, Beaugerie L, Carbonnel F, Parc R, et al. Long-term evolution of disease behavior of Crohn's disease. Inflamm Bowel Dis. 2002;8:244-50.

151. Akobeng AK. The evidence base for interventions used to maintain remission in Crohn's disease. Aliment Pharmacol Ther. 2008;27:11-8.

152. Rutgeerts P, D’Haens G, Targan S, Vasiliauskas E, Hanauer SB, Present $\mathrm{DH}$, et al. Efficacy and safety of retreatment with antitumor necrosis factor antibody (infliximab) to maintain remission in Crohn's disease. Gastroenterology. 1999;117:761-9.

153. Camma C, Giunta M, Rosselli M, Cottone M. Mesalamine in the maintenance treatment of Crohn's disease: a meta-analysis adjusted for confounding variables. Gastroenterology. 1997;113: 1465-73.

154. Lemann M, Mary JY, Colombel JF, Duclos B, Soule JC, Lerebours $\mathrm{E}$, et al. A randomized, double-blind, controlled withdrawal trial in Crohn's disease patients in long-term remission on azathioprine. Gastroenterology. 2005;128:1812-8.

155. Yamamoto T, Nakahigashi M, Saniabadi AR, Iwata T, Maruyama Y, Umegae S, et al. Impacts of long-term enteral nutrition on clinical and endoscopic disease activities and mucosal cytokines during remission in patients with Crohn's disease: a prospective study. Inflamm Bowel Dis. 2007;13:1493-501.

156. Verma S, Kirkwood B, Brown S, Giaffer MH. Oral nutritional supplementation is effective in the maintenance of remission in Crohn's disease. Dig Liver Dis. 2000;32:769-74.

157. Jutta K, Heidi P, Peter L. Management of the short bowel syndrome after extensive small bowel resection. Best Pract Res Clin Gastroenterol. 2004;18:977-92.

158. Yao T, Sakurai T, Hiwatashi N. A study of long-term prognosis of Crohn's disease -cumulative mortality and cumulative rate of surgery. Annual reports of the research group of intractable inflammatory bowel disease subsidized by the Ministry of Health, Labour, and Welfare of Japan, Tokyo, Japan, 1992. p. 49-51 (in Japanese).

159. Cantor M, Bernstein CN. Clinical course and natural history of ulcerative colitis. In: Sartor RB, Sandborn W, editors. Kirsner's inflammatory bowel diseases. Edinburgh: Saunders; 2004. p. $280-88$.

160. Sugita A, Kimura H, Koganei K, Ohtsuki K, Futatsuki R, Fukushima T, et al. Surgical treatment for gastroduodenal Crohn's disease. Stomach Intestine. 2007;42:477-84 (in Japanese).

161. Ulman TA, Itzkowitx SH. Cancer risk in inflammatory bowel disease. In: Satsangi J, Sutherland LR, editors. Inflammatory bowel disease. Philadelphia: Churchill Livingstone; 2003. p. 605-19.

162. Fazio VW, Marchetti F, Church M, Goldblum JR, Lavery C, Hull TL, et al. Effect of resection margins on the recurrence of Crohn's disease in the small bowel. A randomized controlled trial. Ann Surg. 1996;224:563-71 (discussion 571-3).

163. Post S, Herfarth C, Bohm E, Timmermanns G, Schumacher H, Schurmann G, et al. The impact of disease pattern, surgical management, and individual surgeons on the risk for relaparotomy for recurrent Crohn's disease. Ann Surg. 1996;223:253-60.

164. Ozuner G, Fazio VW, Lavery IC, Milsom JW, Strong SA. Reoperative rates for Crohn's disease following strictureplasty. Long-term analysis. Dis Colon Rectum. 1996;39:1199-203. 
165. Sasaki I, Funayama Y, Naito H, Fukushima K, Shibata C, Matsuno S. Extended strictureplasty for multiple short skipped strictures of Crohn's disease. Dis Colon Rectum. 1996;39:342-4.

166. Michelassi F. Side-to-side isoperistaltic strictureplasty for multiple Crohn's strictures. Dis Colon Rectum. 1996;39:345-9.

167. Hughes LE, Taylor BA. Perianal lesions in Crohn's disease. In: Allan RN, Keighley MR, Alexander-Williams J, Hawkins C, editors. Inflammatory bowel disease. Philadelphia: Churchill Livingstone; 1990. p. 351-61.

168. Sugita A. Features and prognosis of small/colorectal cancer complicated with ulerative colitis/Crohn's disease. Annual reports of the research group of intractable inflammatory bowel disease subsidized by the Ministry of Health, Labour, and Welfare of Japan, Tokyo, Japan, 2008. p. 87-9 (in Japanese).

169. Kogenei K, Kimura H, Arai K, Sugita A, Fukushima T. Efficacy and problems of fecal diversion for intractable anorectal complications of Crohn's disease. Jpn J Gastroenterol Surg. 2005;38:1543-48 (in Japanese).

170. Post S, Herfarth C, Schumacher H, Golling M, Schurmann G, Timmermanns G. Experience with ileostomy and colostomy in Crohn's disease. Br J Surg. 1995;82:1629-33.

171. Rutgeerts P, Geboes K, Vantrappen G, Kerremans R, Coenegrachts JL, Coremans G. Natural history of recurrent Crohn's disease at the ileocolonic anastomosis after curative surgery. Gut. 1984;25:665-72.

172. Williams JG, Wong WD, Rothenberger DA, Goldberg SM. Recurrence of Crohn's disease after resection. Br J Surg. 1991;78:10-9.

173. Fukushima K, Sugita A, Baba S. A study of factors for postoperative recurrence of Crohn's disease. Annual reports of the research group of intractable inflammatory bowel disease subsidized by the Ministry of Health, Labour, and Welfare of Japan, Tokyo, Japan, 1996. p. 58-60 (in Japanese).

174. Raab Y, Bergstrom R, Ejerblad S, Graf W, Pahlman L. Factors influencing recurrence in Crohn's disease. An analysis of a consecutive series of 353 patients treated with primary surgery. Dis Colon Rectum. 1996;39:918-25.

175. Greenstein AJ, Lachman P, Sachar DB, Springhorn J, Heimann $\mathrm{T}$, Janowitz HD, et al. Perforating and non-perforating indications for repeated operations in Crohn's disease: evidence for two clinical forms. Gut. 1988;29:588-92.

176. Simillis C, Yamamoto T, Reese GE, Umegae S, Matsumoto K, Darzi AW, et al. A meta-analysis comparing incidence of recurrence and indication for reoperation after surgery for perforating versus nonperforating Crohn's disease. Am J Gastroenterol. 2008;103:196-205.

177. McLeod RS, Wolff BG, Steinhart AH, Carryer PW, O'Rourke $\mathrm{K}$, Andrews DF, et al. Prophylactic mesalamine treatment decreases postoperative recurrence of Crohn's disease. Gastroenterology. 1995;109:404-13.

178. Rutgeerts P, Hiele M, Geboes K, Peeters M, Penninckx F, Aerts $\mathrm{R}$, et al. Controlled trial of metronidazole treatment for prevention of Crohn's recurrence after ileal resection. Gastroenterology. 1995;108:1617-21.

179. Hanauer SB, Korelitz BI, Rutgeerts P, Peppercorn MA, Thisted RA, Cohen RD, et al. Postoperative maintenance of Crohn's disease remission with 6-mercaptopurine, mesalamine, or placebo: a 2-year trial. Gastroenterology. 2004;127:723-9.

180. Matsui T, Seki T, Yao K, Sou S, Hirai F. Diagnosis of inflammatory diseases of the small intestine: comparison between double balloon enteroscopy and double contrast radiography. Stomach Intestine. 2005;40:1491-502 (in Japanese).

181. Consigny Y, Modigliani R, Colombel JF, Dupas JL, Lemann M, Mary JY. A simple biological score for predicting low risk of short-term relapse in Crohn's disease. Inflamm Bowel Dis. 2006;12:551-7.
182. Canavan C, Abrams KR, Mayberry J. Meta-analysis: colorectal and small bowel cancer risk in patients with Crohn's disease. Aliment Pharmacol Ther. 2006;23:1097-104.

183. Jess T, Riis L, Vind I, Winther KV, Borg S, Binder V, et al. Changes in clinical characteristics, course, and prognosis of inflammatory bowel disease during the last 5 decades: a population-based study from Copenhagen, Denmark. Inflamm Bowel Dis. 2007;13:481-9.

184. Masunaga Y, Ohno K, Ogawa R, Hashiguchi M, Echizen H, Ogata $\mathrm{H}$. Meta-analysis of risk of malignancy with immunosuppressive drugs in inflammatory bowel disease. Ann Pharmacother. 2007;41:21-8.

185. Rosh JR, Gross T, Mamula P, Griffiths A, Hyams J. Hepatosplenic T-cell lymphoma in adolescents and young adults with Crohn's disease: a cautionary tale? Inflamm Bowel Dis. 2007;13:1024-30.

186. Friedman S, Rubin PH, Bodian C, Goldstein E, Harpaz N, Present DH. Screening and surveillance colonoscopy in chronic Crohn's colitis. Gastroenterology. 2001;120:820-6.

187. Geborek P, Bladstrom A, Turesson C, Gulfe A, Petersson IF, Saxne T, et al. Tumour necrosis factor blockers do not increase overall tumour risk in patients with rheumatoid arthritis, but may be associated with an increased risk of lymphomas. Ann Rheum Dis. 2005;64:699-703.

188. Wolfe F, Michaud K. The effect of methotrexate and anti-tumor necrosis factor therapy on the risk of lymphoma in rheumatoid arthritis in 19,562 patients during 89,710 person-years of observation. Arthritis Rheum. 2007;56:1433-9.

189. Oriuchi T, Hiwatashi N, Katsurashima Y, Noguchi M, Kinouchi Y, Toyota $\mathrm{T}$. Inflammatory bowel disease and pregnancy: clinical study of mutual influence, clinical course and treatment. Nihon Shokakibyo Gakkai Zasshi. 1999;96:266-72 (in Japanese).

190. Mayberry JF, Weterman IT. European survey of fertility and pregnancy in women with Crohn's disease: a case control study by European collaborative group. Gut. 1986;27:821-5.

191. Moody GA, Probert C, Jayanthi V, Mayberry JF. The effects of chronic ill health and treatment with sulphasalazine on fertility amongst men and women with inflammatory bowel disease in Leicestershire. Int J Colorectal Dis. 1997;12:220-4.

192. Narendranathan M, Sandler RS, Suchindran CM, Savitz DA. Male infertility in inflammatory bowel disease. J Clin Gastroenterol. 1989;11:403-6.

193. Moody GA, Mayberry JF. Perceived sexual dysfunction amongst patients with inflammatory bowel disease. Digestion. 1993;54:256-60.

194. Norgard B, Pedersen L, Christensen LA, Sorensen HT. Therapeutic drug use in women with Crohn's disease and birth outcomes: a Danish nationwide cohort study. Am J Gastroenterol. 2007;102:1406-13.

195. Bortoli A, Saibeni S, Tatarella M, Prada A, Beretta L, Rivolta R, et al. Pregnancy before and after the diagnosis of inflammatory bowel diseases: retrospective case-control study. J Gastroenterol Hepatol. 2007;22:542-9.

196. Dominitz JA, Young JC, Boyko EJ. Outcomes of infants born to mothers with inflammatory bowel disease: a population-based cohort study. Am J Gastroenterol. 2002;97:641-8.

197. Baird DD, Narendranathan M, Sandler RS. Increased risk of preterm birth for women with inflammatory bowel disease. Gastroenterology. 1990;99:987-94.

198. Francella A, Dyan A, Bodian C, Rubin P, Chapman M, Present $\mathrm{DH}$. The safety of 6-mercaptopurine for childbearing patients with inflammatory bowel disease: a retrospective cohort study. Gastroenterology. 2003;124:9-17.

199. Caprilli R, Gassull MA, Escher JC, Moser G, Munkholm P, Forbes $\mathrm{A}$, et al. European evidence based consensus on the 
diagnosis and management of Crohn's disease: special situations. Gut. 2006;55:i36-58.

200. Mahadevan U, Kane S, Sandborn WJ, Cohen RD, Hanson K, Terdiman JP, et al. Intentional infliximab use during pregnancy for induction or maintenance of remission in Crohn's disease. Aliment Pharmacol Ther. 2005;21:733-8.

201. Katz JA, Antoni C, Keenan GF, Smith DE, Jacobs SJ, Lichtenstein GR. Outcome of pregnancy in women receiving infliximab for the treatment of Crohn's disease and rheumatoid arthritis. Am J Gastroenterol. 2004;99:2385-92.

202. Present DH. Pregnancy and inflammatory bowel disease. In: Bayless TM, Hanauer SB, editors. Advanced therapy of inflammatory bowel disease. Shelton: PMPH-USA; 2001. p. 613-8.

203. Kinjo F, Chinen H. Issues of pregnancy and delivery in IBD. IBD Res. 2008;2:61-6 (in Japanese).

204. Miller LG, Hopkinson JM, Motil KJ, Corboy JE, Andersson S. Disposition of olsalazine and metabolites in breast milk. J Clin Pharmacol. 1993;33:703-6.

205. Esbjorner E, Jarnerot G, Wranne L. Sulphasalazine and sulphapyridine serum levels in children to mothers treated with sulphasalazine during pregnancy and lactation. Acta Paediatr Scand. 1987;76:137-42. 